\title{
1 The trajectory of discrete gating charges in a voltage-gated potassium channel
}

3 Michael F. Priest ${ }^{1,3}$, Elizabeth E.L. Lee ${ }^{1,3}$, Francisco Bezanilla ${ }^{1,2, *}$

$4{ }^{1}$ Committee on Neurobiology and Department of Biochemistry and Molecular Biology,

5 University of Chicago, Chicago, IL

$6{ }^{2}$ Centro Interdisciplinario de Neurociencia de Valparaíso, Facultad de Ciencias, Universidad de

7 Valparaíso, Valparaíso, Chile

$8 \quad 3$ These authors contributed equally

9 * Correspondence: fbezanilla@uchicago.edu

\section{Abstract}

12 Positively-charged amino acids respond to membrane potential changes to drive

13 voltage sensor movement in voltage-gated ion channels, but determining the trajectory

14 of voltage sensor gating charges has proven difficult. We optically tracked the

15 movement of the two most extracellular charged residues $(\mathrm{R} 1, \mathrm{R} 2)$ in the Shaker

16 potassium channel voltage sensor using a fluorescent positively-charged bimane

17 derivative $(\mathrm{qBBr})$ that is strongly quenched by tryptophan. By individually mutating

18 residues to tryptophan within the putative trajectory of gating charges, we observed that

19 the charge pathway during activation is a rotation and a tilted translation that differs

20 between R1 and R2 and is distinct from their deactivation pathway. Tryptophan-induced

21 quenching of $\mathrm{qBBr}$ also indicates that a crucial residue of the hydrophobic plug is linked

22 to the Cole-Moore shift through its interaction with R1. Finally, we show that this

23 approach extends to additional voltage-sensing membrane proteins using the Ciona

24 intestinalis voltage sensitive phosphatase (CiVSP). 


\section{Introduction}

27 The nature of the motion of the voltage sensor in voltage-gated ion channels has been a

28 subject of intensive research. This motion is driven by voltage changes sensed by

29 positively-charged amino acids (typically arginines) found on the fourth transmembrane

30 segment (S4) of each monomer of the tetrameric channel. The number of charged

31 amino acids driving this motion varies from channel to channel, but has been shown in

32 the canonical voltage-gated Shaker potassium channel $(\mathrm{Kv})$ to consist of the four most

33 extracellular arginines (Aggarwal \& MacKinnon, 1996; Seoh et al., 1996). However,

34 research into the motion of these individual gating charges has suffered from

35 experimental limitations. Replacement of arginine by histidine together with $\mathrm{pH}$ titration

36 allows the study of gating charge end positions during activation, but does not show the

37 charge's actual trajectory (Starace et al., 1997; Starace \& Bezanilla, 2001). Cross-

38 linking studies replace residues with cysteines to see which residues interact with each

39 other in different conformational states, necessarily abrogating the positive charge of

40 any gating charge if they are examined directly (Broomand et al., 2003; Henrion et al.,

41 2012; Lainé et al., 2003), and accessibility studies have the same limitation (Yang \&

42 Horn, 1995). Similarly, site directed fluorimetric approaches typically replace a residue

43 with a cysteine and then attach a fluorescent dye, providing the additional advantage of

44 being able to monitor conformational changes in real-time, but do not directly follow the

45 movement of the gating charges (Cha \& Bezanilla, 1997; Mannuzzu et al., 1996; Priest

46 \& Bezanilla, 2015). Positively charged adducts such as methanethiosulfonate-

47 ethyltrimethylammonium (MTSET) linked to cysteines placed at gating charges can be 
used to replace the positive charge and characterize discrete gating charges (Ahern \& Horn, 2004, 2005; Baker et al., 1998; Larsson et al., 1996). However, these replacement charges cannot be rapidly monitored, limiting their use for observing

51 conformational changes.

Ideally, one would like to follow the movement of individual gating charges in real

53 time as they respond to changes in the electric field. This requires a fluorophore that is

54 comparable to the gating arginines. We used monobromo(trimethylammonio)bimane

55 (qBBr), a small molecule fluorescent dye with a permanent positive charge (Figure 1A).

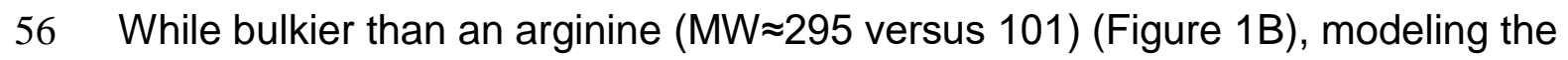

57 conjugation of $\mathrm{qBBr}$ to a cysteine substituted into the two most extracellular gating

58 charges in a Shaker-type voltage-gated potassium channel (R362 or R1 and R365 or

59 R2) (Chen et al., 2010; Pettersen et al., 2004) provided a distance between the carbon

60 backbone and the positive charge of $\mathrm{qBBr}$ of $\sim 5.5 \AA$, very close to the analogous

61 distance of $\sim 5.3$ to $6.5 \AA$ for arginine. Therefore, $\mathrm{qBBr}$ attached to a cysteine at the

62 endogenous position of the gating charge may mimic the positive gating charge. In

63 addition to being positively charged, $\mathrm{qBBr}$ has useful fluorescence quenching properties.

64 Tryptophan has been shown to strongly quench qBBr fluorescence, with weak

65 quenching by tyrosine, and no quenching from various other amino acids including

66 histidine, phenylalanine, methionine, aspartate, or arginine (Mansoor et al., 2010). This

67 phenomenon of 'tryptophan-induced quenching' in bimane dyes generally (Mansoor et

68 al., 2002), together with their remarkable environmental insensitivity (Mansoor et al.,

69 1999), has been taken advantage of to measure conformational rearrangements of

70 various membrane proteins, including the $\beta 2$-adrenergic G protein-coupled receptor 
71 (Yao et al., 2006), a cyclic nucleotide-gated ion channel (Islas \& Zagotta, 2006), the BK

72 channel (Semenova et al., 2009), a proton-gated ion channel (Menny et al., 2017), and

73 a lactose permease (Smirnova et al., 2014). In general, these studies use the

74 fluorescent quenching produced by an interaction between bimane and a particular

75 tryptophan, whether native or engineered into the protein, to provide insight into how the

76 protein moves during conformational transitions.

Here, we perform site-directed voltage-clamp fluorimetry on qBBr-bound Shaker

$78 \mathrm{Kv}$ channels expressed in Xenopus laevis oocytes. By substituting a cysteine for a

79 native gating charge and then covalently attaching $\mathrm{qBBr}$ to this site, we produce a

80 fluorescent mimic of a discrete charge in the voltage sensor of the channel. Individual

81 native or engineered tryptophans in the channel are then used to quench the

82 fluorescence of the charged $\mathrm{qBBr}$ as it moves in response to changes in membrane

83 potential. We used this system to investigate the pathway taken by R1 and R2 during

84 activation and deactivation, as well as to examine the effect of the relaxed state (Lacroix

85 et al., 2011; Villalba-Galea et al., 2008) on these individual gating charges. In general,

86 the movement of $\mathrm{R} 1 \mathrm{C}$ - and $\mathrm{R} 2 \mathrm{C}-\mathrm{qBBr}$ follow a tilted translation across the membrane

87 and rotation, giving new information on the trajectories that have been inferred from

88 recent consensus models on the motion of the voltage sensor (Li et al., 2014; Vargas et

89 al., 2011, 2012). Of particular interest, during normal channel activation R1C-qBBr does

90 not appear to interact with F290W, the most intracellular residue of the hydrophobic

91 plug (Chen et al., 2010; Lacroix \& Bezanilla, 2011), also called the gating charge

92 transfer center (Tao et al., 2010). Instead, R1 only interacts with F290W at extremely

93 negative potentials, suggesting that R1 does not normally move past the hydrophobic 
94 plug and provides the basis of the Cole-Moore shift. This technique should also be

95 transferrable to other voltage-sensing membrane proteins; as a proof of principle, we

96 demonstrate its use in the voltage-sensitive phosphatase CiVSP.

97

$98 \underline{\text { Results }}$

99 The basic principles of qBBr gating charge tracking in time

100 The idea of the present approach is to study the translocation of the gating

101 charge (now a fluorophore, $\mathrm{qBBr}$ ) as the membrane potential is changed using the

102 specific quenching of qBBr by a tryptophan $(\mathrm{W})$ that is positioned nearby or in the path

103 of qBBr.

104 If we were tracking fluorescence at the single molecule level, the fluorescence

105 signal we would observe would depend on the position of the $\mathrm{W}$ with respect to the

106 moving $\mathrm{qBBr}$. Let us assume that we are applying a positive voltage to activate the

107 voltage sensor that moves between two discrete positions. We can distinguish three

108 extreme cases schematically (Figure 1C, left panel). If the $\mathrm{W}$ is near the resting position

109 of the qBBr, we would see a sudden increase in fluorescence when the qBBr moves

110 away from it and the time lag before that increase corresponds to the waiting time of the

111 sensor before it jumps across the energy barrier (Figure 1C, left, top trace). On the

112 other hand, if the $\mathrm{W}$ is in the path of $\mathrm{qBBr}$ (Figure $1 \mathrm{C}$, left, middle), we would see an

113 extremely brief decrease of fluorescence as the qBBr passes by the quenching group.

114 Finally, if the $\mathrm{W}$ is near the final position of the $\mathrm{qBBr}$ (Figure $1 \mathrm{C}$, left, bottom) we would

115 see a sudden decrease in fluorescence that would be maintained when the $\mathrm{qBBr}$ 
116 reaches that point. The duration of the high fluorescence period is the waiting time

117 before the sensor crosses the energy barrier.

118 However, since we are looking at a large ensemble of molecules the

119 macroscopic fluorescence signals we see are the result of an ensemble of voltage-

120 sensing domains (VSDs) moving, each one at a different time according to its first

121 latency duration, generating a continuous change in fluorescence. The fast event for the

122 case of a W in the middle of the qBBr path would not be visible in the ensemble

123 because the crossing of the VSDs is unsynchronized and if it is exactly in the middle no

A<smiles></smiles>

B

124

125 Figure 1. The basic principles of $\mathrm{qBBr}$ gating charge tracking in time

126 (A) Chemical structure of monobromo(trimethylammonio)bimane, a small positively charged

127 (blue) fluorophore with the ability to conjugate to cysteine (yellow). (B) Size comparison of $\mathrm{qBBr}$ -

128 Cys (top) to an arginine (bottom). (Blue, nitrogen; red, oxygen; sulfur, yellow; grey, carbon). (C)

129 Cartoon schematic of qBBr-tryptophan distance-based quenching with representative

130 fluorescence data for unquenching (top), no effect (center), and quenching (bottom). 
132 signal will be seen (Figure 1C, right panel, middle trace). The other two extreme cases

133 will generate a continuous quenching or unquenching depending on the placement of

134 the tryptophan (Figure 1C, right, top and bottom).

137 A simple model of quenching of qBBr by $\mathrm{W}$ is

$$
F(t)=F_{o}\left[1-\exp \left(-\frac{d}{\lambda}\right)\right]
$$

where $F(t)$ is qBBr fluorescence, $F_{0}$ is the maximum qBBr fluorescence (no quenching),

$141 d$ is the distance from $\mathrm{qBBr}$ to $\mathrm{W}$ and ? is the characteristic distance for quenching, Now,

142 we assume that qBBr moves in a trajectory represented by $x$ between the initial position,

$143 \mathrm{X} 0$, and the final position, $\mathrm{Xf}_{\mathrm{f}}$, and $\mathrm{W}$ is located somewhere near the $\mathrm{x}$ trajectory, $\mathrm{Xw}$. Let

144 us consider first that qBBr moves between two states and define $P_{0}(t)$ and $P_{f}(t)$ as the

145 probabilities that $\mathrm{qBBr}$ is in $x_{0}$ and $x_{f}$ respectively. Then, $P_{0}(t)+P_{f}(t)=1$ and we can write 146 the time course of the fluorescence as

$$
\frac{F(t)}{F_{0}}=P_{0}(t)\left[\exp \left(-\frac{\left|x_{w}-x_{f}\right|}{\lambda}\right)-\exp \left(-\frac{\left|x_{w}-x_{0}\right|}{\lambda}\right)\right]+1-\exp \left(-\frac{\left|x_{w}-x_{f}\right|}{\lambda}\right)
$$

150 This means that the time course of fluorescence will have the time course of $P_{0}(t)$ and

151 will not depend on the position of $\mathrm{W}$ in the trajectory.

152 If we now assume that the number of states is more than 2 , say $i=1,2,3, \ldots, n$, we can

153 write a general solution for the fluorescence 


$$
\frac{F(t)}{F_{0}}=\sum_{i=1}^{n} P_{i}(t)\left[1-\exp \left(-\frac{\left|x_{i}-x_{w}\right|}{\lambda}\right)\right]
$$

$155 P_{i}(t)$ will have $n-1$ eigenvalues, so the time course of fluorescence will now depend on

156 the position of the $\mathrm{W}$ in the trajectory, because $P_{i}(t)$ will be weighted by the exponential

157 term that includes the distance to each position. Simulations with 3 states show

158 fluorescence time courses with more than one exponential component and, depending 159 on the position and dwell time in the intermediate state, the response may be biphasic

160 (i.e. fluorescence increases and decreases) during the depolarization. This is expected

161 because as it traverses its trajectory the qBBr will go through periods when it will be 162 closer to the $\mathrm{W}$ and others when it will be further away. This is a prediction that will be 163 important in interpreting the experimental results.

\section{qBBr mimics a native gating charge}

While qBBr crucially contains a permanent charge (Figure $2 \mathrm{~A}$ ) like the arginines

167 that constitute the gating charges in the Shaker Kv channel, this is not a guarantee that

168 the voltage sensor will continue to behave normally with a fluorescent dye substituted

169 for a gating charge. To test whether this was the case, we first separately mutated R1

170 and $\mathrm{R} 2$ in a non-conducting (W434F), non-fast inactivating ( $\Delta 6-46)$ Shaker channel to

171 cysteines. Following expression of these constructs in Xenopus laevis oocytes, $\mathrm{qBBr}$

172 was conjugated to either of these charges by incubation of the oocytes in a depolarizing

173 solution containing $\mathrm{qBBr}$. Oocytes containing $\mathrm{qBBr}$ conjugated to $\mathrm{R} 1 \mathrm{C}$ or $\mathrm{R} 2 \mathrm{C}$ were

174 then simultaneously recorded optically and electrophysiologically using the cut-open

175 oocyte voltage clamp technique. In both cases, the observed gating currents are likely a 
177 sensors that retain a bare cysteine. For R1C-qBBr, gating currents appeared to be

178 faster than those of the wild type channel but still comparable in voltage sensitivity

179 (Figures 2A and 2B). With R2C-qBBr, gating currents were also more rapid, with voltage

180 sensor movement beginning at more hyperpolarized potentials than in the wild-type

181 channel and producing a shallow $\mathrm{Q}-\mathrm{V}$ curve (Figures $2 \mathrm{~A}$ and $2 \mathrm{~B}$ ). These findings

182 suggest that the additional bulk of qBBr may destabilize the resting state of the voltage

183 sensor when it is conjugated to the more intracellular $\mathrm{R} 2 \mathrm{C}$, but not when it is conjugated

184 to the more extracellular R1C. This is in good agreement with numerous computational

185 models of the resting state of the $\mathrm{Kv}$ channel that suggest that R1 is less sterically

186 inhibited in the resting state than R2 (Vargas et al., 2012). They also suggest that $\mathrm{qBBr}$

187 would act as a faithful mimic of the movement of the gating charge of R1, and should

188 also mimic the movement of R2, albeit with energetic differences.

189 Upon depolarization, the fluorescence signal of R1C-qBBr decreased

190 dramatically (Figure 2C, top), while the corresponding fluorescence signal of $\mathrm{R} 2 \mathrm{C}-\mathrm{qBBr}$

191 did not (Figure 2C, bottom). A reduction in qBBr fluorescence is likely due to the

192 presence of a tryptophan, or possibly a tyrosine. Since the fluorescence signal is lower

193 when the $\mathrm{R} 1 \mathrm{C}-\mathrm{qBBr}$ is in the active state than when it is in the resting state, the

194 quenching residue should be near the active state of R1. Based on a homology model

195 of a Kv channel crystal structure in the relaxed state (Chen et al., 2010), R1 should 
A
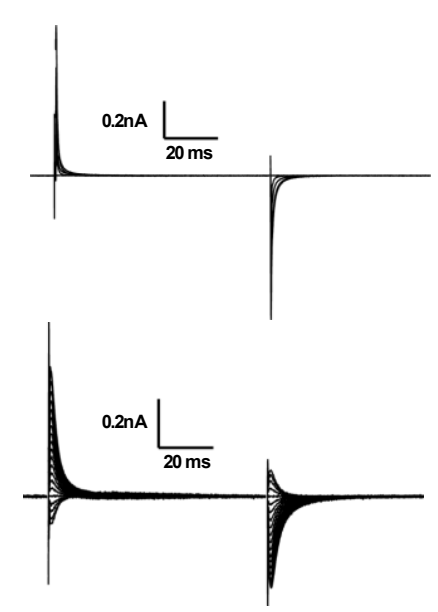

196

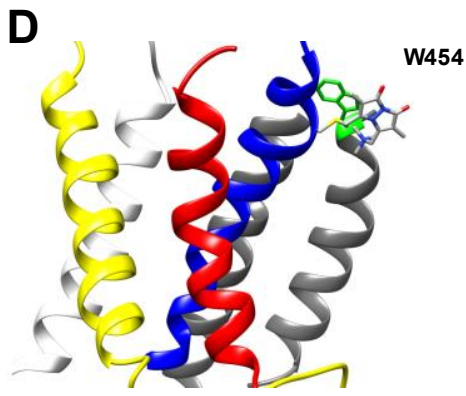

B
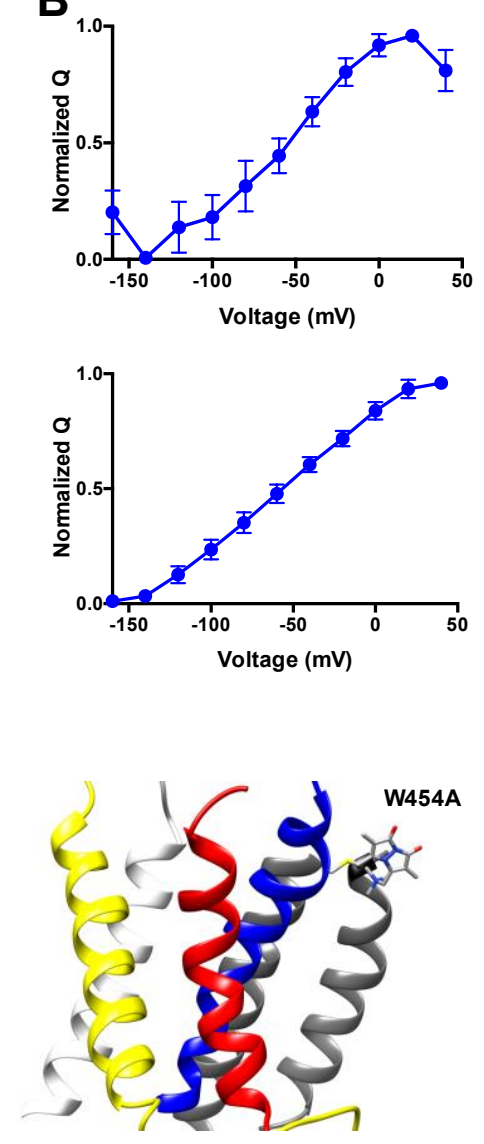

C
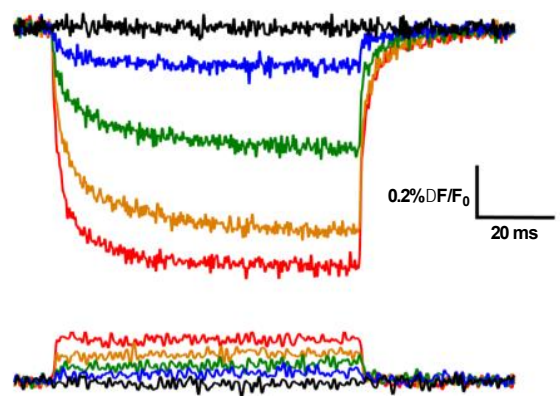

E

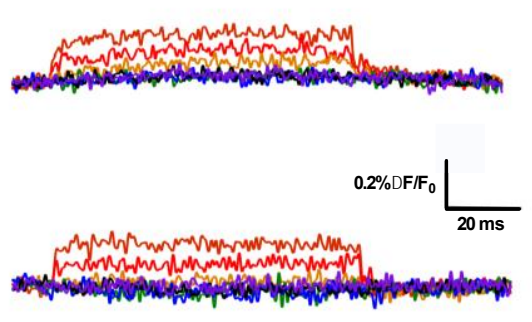

Figure 2. $\mathrm{qBBr}$ mimics a native gating charge

(A) Representative gating current traces of Shaker R1C-qBBr (top) and R2C-qBBr (bottom). (B) Normalized charge $(\mathrm{Q})$ versus voltage $(\mathrm{V}) \mathrm{QV}$ curves of R1C-qBBr (top, $\mathrm{n}=4$ ) and R2C-qBBr (bottom, $n=3$ ). Data are shown as mean \pm SEM. (C) Representative fluorescence traces of $q B B r$ labeled $\mathrm{R} 1 \mathrm{C}-\mathrm{qBBr}$ (top) and $\mathrm{R} 2 \mathrm{C}-\mathrm{qBBr}$ (bottom). In all figures, membrane potentials during the pulse are: brown, $+80 \mathrm{mV}$; red, $+40 \mathrm{mV}$; orange, $0 \mathrm{mV}$; green, $-40 \mathrm{mV}$; blue, $-80 \mathrm{mV}$; black, $120 \mathrm{mV}$; purple, $-160 \mathrm{mV}$. (D) Structures showing the position of the in-silico mutation 454 from a tryptophan (left) to an alanine (right) (PDB: 3LUT). In all Shaker structures, transmembrane domains (S1-S6) are colored: S1, white; S2, yellow; S3, red; S4, blue; S5 and S6, grey. (E) Representative fluorescence traces for R1C-qBBr:W454A (top) and R1C-qBBr:W454F (bottom). 
209 reside near an endogenous tryptophan (W454) at the extracellular side of S6 (Figure

210 2D). Additionally, it has been reported that R1 comes into close proximity to this region

211 of the channel (Lainé et al., 2003). Mutation of this tryptophan to a non-quenching

212 alanine abolished the fluorescence reduction that we observed during activation (Figure

$2132 \mathrm{E}$, top). The mutation of this tryptophan to a phenylalanine also abolished the

214 endogenous voltage-dependent fluorescence (Figure 2E, bottom). However, as this

215 mutation reduced expression, we proceeded with a W454A background. Therefore, our

216 optical data supports the idea that upon activation, $\mathrm{R} 1 \mathrm{C}-\mathrm{qBBr}$ comes near the pore

217 domain where its fluorescence is quenched by W454, while R2C-qBBr does not.

218 These results are in agreement with crystallographic and functional data and suggest

219 that $\mathrm{R} 2 \mathrm{C}-\mathrm{qBBr}$ and $\mathrm{R} 1 \mathrm{C}-\mathrm{qBBr}$ may mimic the motion of the native gating charges.

221 An exogenously substituted tryptophan (E247W) produces voltage-dependent

\section{2 fluorescence changes in R1C-qBBr}

To map the trajectory of $\mathrm{R} 1$ and $\mathrm{R} 2$ using $\mathrm{qBBr}$, we created a series of constructs

224 based on the R1C:W454A and the R2C backgrounds. We used these two constructs as

225 backgrounds because they only showed a very small depolarization-induced increase in

226 fluorescence that had a fast time course with no voltage dependent kinetics (Figure 2E)

227 which we were unable to link to any endogenous tyrosine or tryptophan (Figure 2-figure

228 supplement 1). As this residual fluorescence change was unobservable in the presence

229 of tryptophan-induced quenching in the R1C-qBBr:W454W construct, we hypothesized

230 that substituting a tryptophan elsewhere into the channel would similarly alter the $\mathrm{qBBr}$

231 fluorescence signal in a way that would dominate over the residual fluorescence signal. 
As an example, we substituted a tryptophan at position 247 of the R1C-W454A

233 construct, generating R1C-qBBr:W454A;E247W (Fig. 3A). In response to changes in

234 membrane potential, the exogenous, substituted tryptophan produced voltage-induced

235 fluorescence changes (Figure 3B) that were markedly different from the background

236 fluorescence changes of $\mathrm{R} 1 \mathrm{C}-\mathrm{qBBr}: W 454 \mathrm{~A}$ (Figure 2E, top). If R1C-qBBr is closer to

237 the tryptophan at 247 in the resting state than in the active state, we should observe an

238 increase in fluorescence upon activation (Figure 1C, top). The experiment shows that

239 during activation, E247W produces a clear unquenching, or increase in fluorescence, of

240 R1C-qBBr (Figure 3B). Therefore, R1C-qBBr moves away from position 247 upon

241 activation.

242 Examining the fluorescence signal more closely, we see that it has many

243 properties reflecting movement of the voltage sensor. Just as the gating currents

244 produced by the voltage sensor are voltage-sensitive, the fluorescence signal of R1C-

245 qBBr:W454A;E247W has a voltage-sensitive response. From our family of fluorescence

246 traces (Figure 3B) we can generate a change in fluorescence versus voltage (FV) curve

247 and observe the amount of fluorescence quenching or unquenching due to changes in

248 voltage (Figure 3C). Moreover, the voltage-sensitivity of the fluorescence is also seen in

249 the kinetics of the fluorescence traces (Fig. 3D).

251 Activation pathways mapped by $\mathrm{qBBr}$ and substituted tryptophan-induced

252 fluorescence quenching

253 We generated numerous constructs with an exogenous, substituted tryptophan that 254 produced voltage-induced fluorescence changes in $\mathrm{R} 1 \mathrm{C}-\mathrm{qBB}: \mathrm{W} 454 \mathrm{~A}$ or $\mathrm{R} 2 \mathrm{C}-\mathrm{qBBr}$ 
A

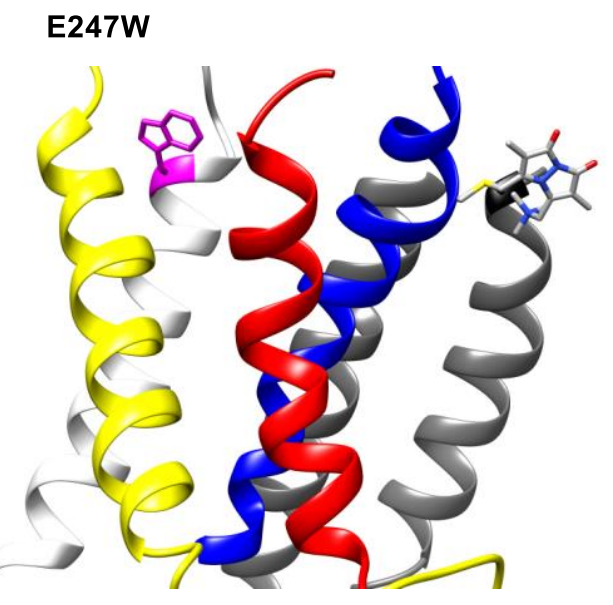

C

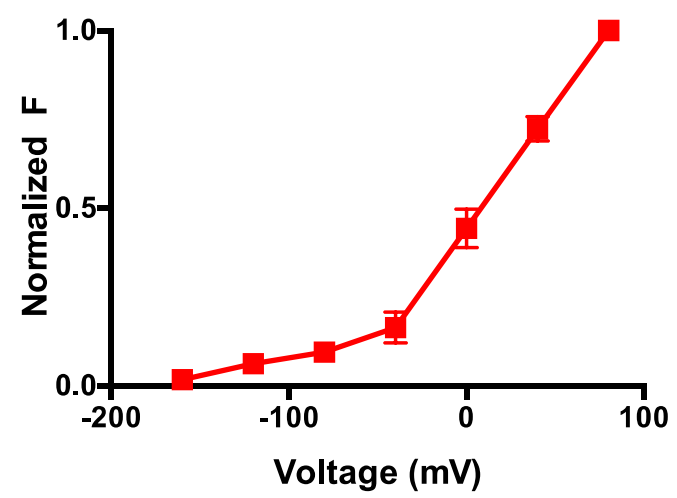

B

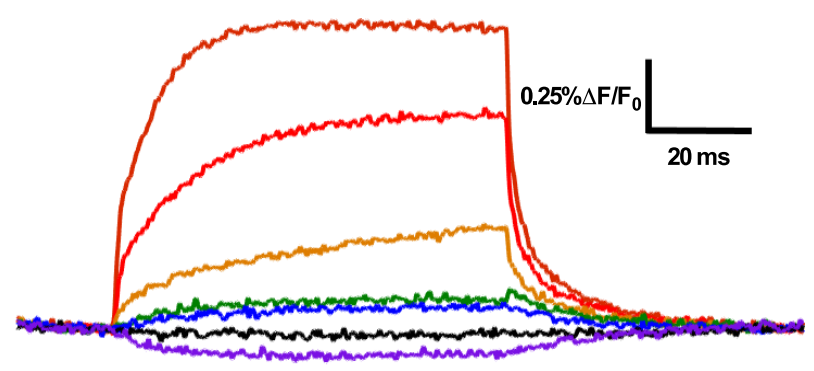

D

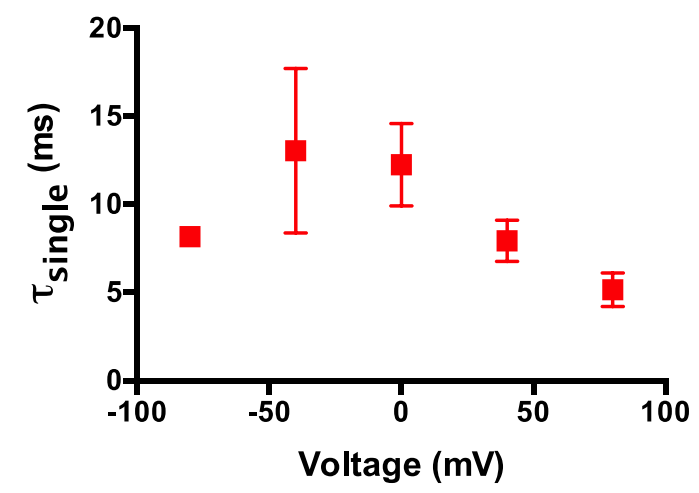

Figure 3. An exogenously substituted tryptophan (E247W) produces voltage-dependent

(A) Homology structure of Shaker construct, R1C-qBBr:W454A;E247W demonstrating the traces. (C) A normalized fluorescence vs voltage curve. (D) A single exponential $\boldsymbol{\tau}$ of

261 fluorescence upon activation $(n=3)$. Data are shown as mean \pm SEM.

263 that were distinct from the background fluorescence changes and from each other

264 (Figures 3B, 3C, 4A and 4B). A decrease in fluorescence upon depolarization suggests

265 that the R1C- or R2C-qBBr comes closer to the inserted tryptophan residue during the

266 transition from the resting to the active state, while an increase in fluorescence upon 
267 depolarization suggests that the gating charge moves away from that residue during

268 activation (Figure 1C). Therefore, by pooling the data from numerous constructs, each

269 with a single tryptophan, we can map the activation pathways of R1 and R2 (Figures 4A

270 and $5 A)$.

In response to a depolarizing pulse, $\mathrm{R} 1 \mathrm{C}-\mathrm{qBB}$ fluorescence was

272 quenched by the endogenous tryptophan at residue 454, as well as by a tryptophan

273 substituted for the phenylalanine at the extracellular side of the S5 at position 416 . R1C-

274 qBBr moved away from tryptophans inserted at F244W, E247W, 320W, and T326W

275 (Figures 4A and 4B). Interestingly, we found no appreciable changes in the

276 fluorescence signal of F290W or L294W in response to depolarizing pulses (Figures 4A

277 and 4B). The small fast fluorescence signal seen for these two constructs is reminiscent

278 of that of R1C-qBBr:W454A, (Figure 2E and Figure 2-figure supplement 1), i.e., the

279 residual fluorescence signal that is small and has fast voltage-independent kinetics.

280 Together these findings map out a pathway for R1 that consists of both an intracellular

281 to extracellular translation, tilted by about $30^{\circ}$ with respect to the normal of the

282 membrane plane, and a rotation (Fig. 4B). Specifically, R1C-qBBr seems to reside near

283 the pore domain in the active state, as observed in the crystal structure (Chen et al.,

284 2010). It reaches this position from a resting position that seems to reside approximately

285 one helix turn extracellular to F290, as it shows movement away from I320W, but not

286 from W289W, F290W, or L294W.

287 Substitution of $\mathrm{W}$ residues along the pathway of $\mathrm{R} 2$ reveals that upon activation

288 R2C-qBBr moves closer to Y415W, F416W, and T326W, while moving away from

289 I241W, F244W, I287W, and L294W (Figures 5A and B). Therefore, in the active state, 
A

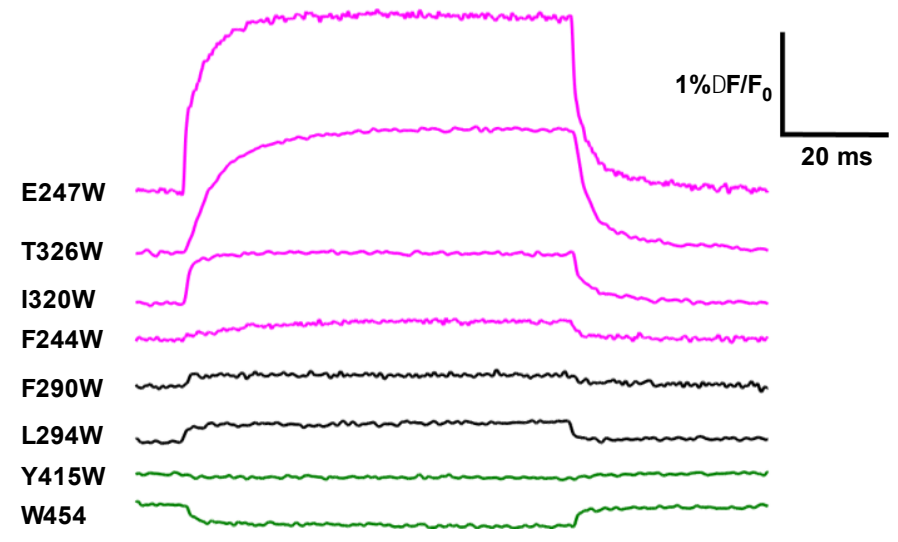

292 substituted tryptophans.

293 (A) Representative $\mathrm{qBBr}$ traces at $+80 \mathrm{mV}$. As $\mathrm{qBBr}$ moves closer to a $\mathrm{W}$, the $\mathrm{W}$ quenches

294 (green) the qBBr fluorescence (Y415W, W454). When it moves further away from a W, the qBBr

295 fluorescence is unquenched (pink; F244W, E247W, I320W, T326W). Some W mutations have

296 no effect (black; 289W, L294W, F290W). (B) A summary structure of activation of R1C-qBBr,

297 with side (left) and extracellular (right) views of the VSD. The activation pathway based on the

298 W quenching/unquenching for R1C-qBBr is marked by a grey arrow.

$300 \mathrm{R} 2 \mathrm{C}-\mathrm{qBBr}$ resides between the extracellular side of the S3 and S5 segments, as

301 quenching is observed from T326W, Y415W, and F416W. As opposed to R1C-qBBr, 
$\mathrm{R} 2 \mathrm{C}-\mathrm{qBBr}$ does not encounter the $\mathrm{S} 6$ segment upon activation. In the resting state, the

303 fluorescence results suggest that R2C-qBBr is stable near F290, unquenching from a

A
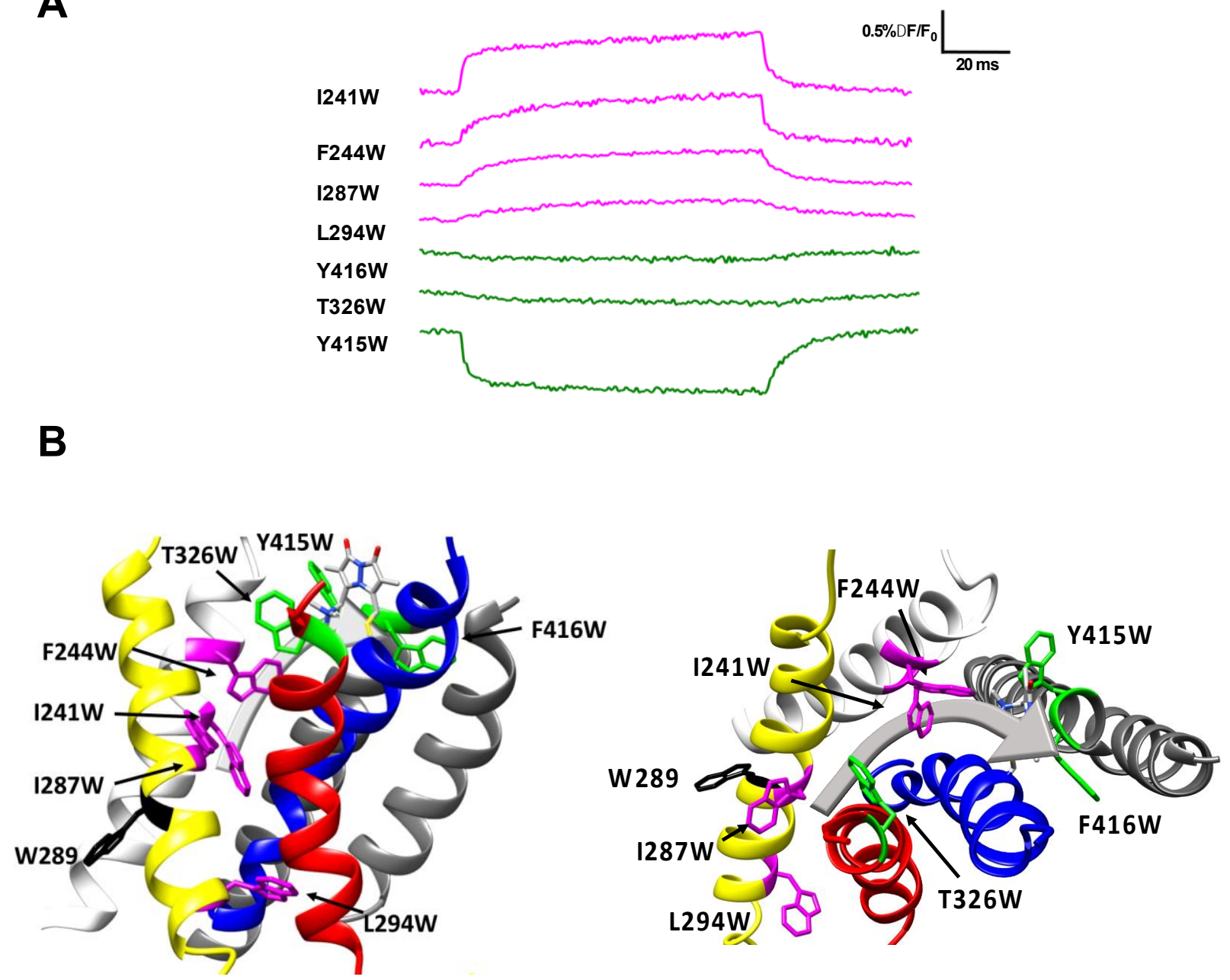

Figure 5. Activation pathway of R2C-qBBr mapped by several individually substituted tryptophans.

307 (A) Representative $\mathrm{qBBr}$ traces at $+80 \mathrm{mV}$. As $\mathrm{qBBr}$ moves closer to a $\mathrm{W}$, the $\mathrm{W}$ quenches

308 (green) the qBBr fluorescence (Y415W, T326W, Y416W). When it moves further away from a W,

309 the qBBr fluorescence is unquenched (pink; I241W, F244W, I287W, L294W). Residue W289

310 (black) does not affect the fluorescence. (B) A summary structure of activation of R2C-qBBr with

311 side (left) and extracellular (right) views of the VSD. The activation pathway based on the W

312 quenching/unquenching for R2C-qBBr is marked by a grey arrow. 
313 cluster of tryptophans inserted at L294, I287, and I241. As seen with R1, there is a

314 rotation in the movement of $\mathrm{R} 2$; however, the rotation of $\mathrm{R} 2$ is less evident.

315 Interestingly, the fluorescence responses differ qualitatively between the two

316 gating charges for tryptophans substituted at L294, T326, and W454. Thus, qBBr

317 mapping reveals that $\mathrm{R} 1$ and $\mathrm{R} 2$ travel distinct paths in relationship to the voltage-

318 sensing domain during activation. Notable exceptions to this include Y415, W289, and

319 F244, which produce similar responses from both R1C and R2C-qBBr. As it faces the

320 lipid membrane, it is unsurprising that W289 does not quench either R1C or R2C-qBBr;

321 however, this suggests that the S2 in which it resides is unlikely to undergo any large

322 rotations that would expose this residue to the gating charges. Y415W quenches both

$323 \mathrm{R} 1$ and R2, underscoring the activated position of both these residues near the

324 extracellular surface of the membrane. The neighboring residue F416W quenched R2C-

$325 \mathrm{qBBr}$ and did not express with R1C-qBBr. F416 has previously been shown to bridge to

326 R1 (Conti et al., 2016; Lainé et al., 2003; Phillips \& Swartz, 2010) and R2 (Conti et al.,

327 2016). Finally, F244 has been shown to interact with both R1 and R2 (Lacroix et al.,

328 2012), and has been proposed to act as a critical stabilizer of the active state of the

329 voltage sensor (Lacroix et al., 2014). Together, our fluorescence data show that upon

330 activation both $\mathrm{R} 1$ and $\mathrm{R} 2$ undergo a tilted translation from the intracellular to the

331 extracellular side of the membrane, together with a rotation (Figures 4B and 5B). The

332 discrete and unique pathways mapped out by these charges provide new levels of

333 clarity into how these charges move distinctly from each other. 
336 Previous studies have discussed the asymmetry in activation and deactivation currents,

337 with deactivation currents being slower than those of activation (Labro et al., 2012;

338 Lacroix et al., 2011; Lacroix \& Bezanilla, 2012). Specifically, deactivation currents are

339 slowed down in a voltage-dependent manner and correspond with pore opening

340 (McCormack et al., 1994; Perozo et al., 1993). If the path of deactivation was the same

341 as that of activation, we would expect $\mathrm{qBBr}$ fluorescence to display similar voltage

342 dependence and kinetics during both activation (Figure 6A, left) and deactivation

343 (Figure 6A, right). The comparison of the activation charge versus voltage curves (QVs)

344 and FVs to the deactivation QVs and FVs shows that during deactivation there are

345 leftward shifts in several R1C-qBBr:W454A constructs: E247W, I320W, and T326W

346 (Figures 6B and C). The shifts in QV and FV curves indicate that the VSD has a

347 different energetic path from the active to the resting state than from the resting state to

348 the active state. This is also reflected in the speed of the fluorescence traces, where we

349 do not see exact overlap in the kinetics of activation and deactivation fluorescence

350 (Figure 6D). In two of our constructs, R1C-qBBr:W454A;E247W and R1C-

351 qBBr:W454A;I320W, the kinetics of the deactivation fluorescence are slower than that

352 of activation. However, we detect the opposite for the fluorescence kinetics of R1C-

353 qBBr:W454A;T326W (Figure 6D, right) where activation fluorescence kinetics are

354 slower than those of deactivation. Others have observed that with the T326W mutation,

355 both activation and deactivation are slowed, but activation much more strongly (Hong \&

356 Miller, 2000). Thus, we have strong evidence through both the differing kinetics and the

357 leftward shifts in the FVs of R1C-qBBr fluorescence that a discrete gating charge takes 
A

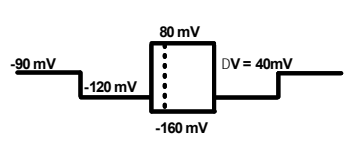

E247W

B

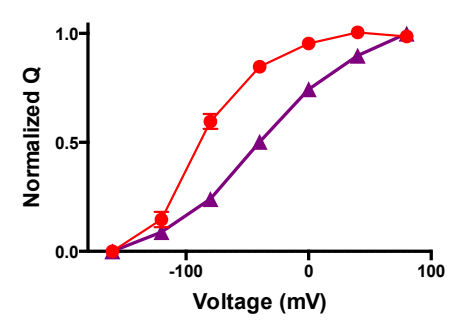

C

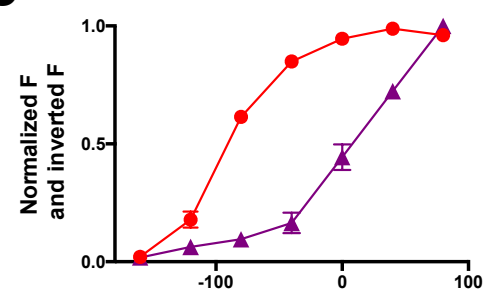

D

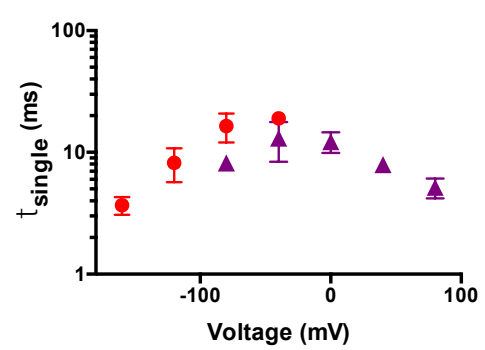

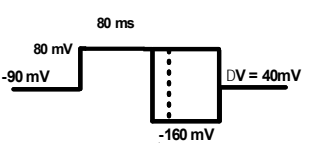

I320W
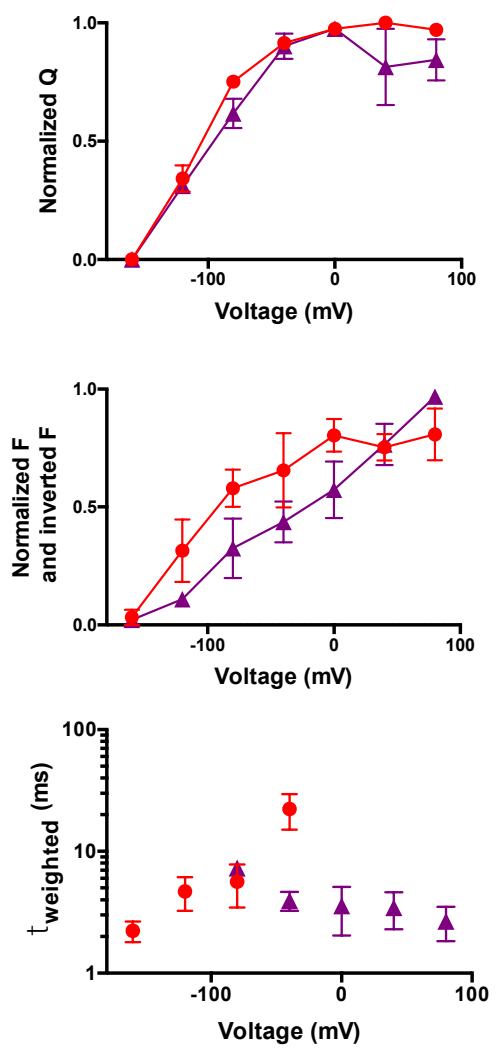

$\Delta$ Activation

- Deactivation

Figure 6. The VSD deactivation path differs from that of activation

360 (A) Activation (purple) and deactivation (red) protocols. Shading indicates where gating and

361 fluorescence were measured for (B-D). (B) Comparison of activation (purple triangles) and

362 deactivation (red circles) QVs for R1C-qBBr:W454A;E247W (left, $n=8$ ), R1C-

363 qBBr:W454A;I320W (center, n=5), and R1C-qBBr:W454A;T326W (right, $n=7$ ). (C) As in (B), but

364 a comparison of activation and deactivation FVs, rather than QVs. (D) Comparison of single

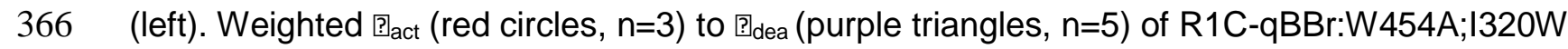

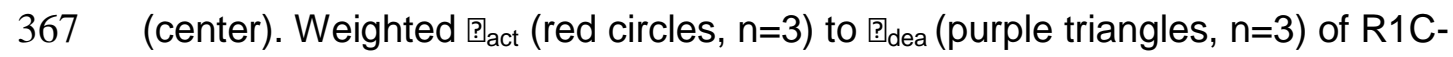


369 a different path in activation than during deactivation; this is also seen with $\mathrm{R} 2 \mathrm{C}-\mathrm{qBBr}$

370 (Figure 6-figure supplement 1).

371 In addition to a resting state and an active state, the voltage sensor has a third

372 state, called the relaxed state, which it enters after prolonged depolarization ( $\mathrm{F}$.

373 Bezanilla et al., 1982; Lacroix et al., 2011; Villalba-Galea et al., 2008). This state has

374 been observed in most S4-based voltage sensors, including voltage-gated sodium

375 channels (Nav), Kv, hyperpolarization-activated cyclic nucleotide-gated channels (HCN),

376 and CiVSP (F. Bezanilla et al., 1982; Bruening-Wright \& Larsson, 2007; Villalba-Galea

377 et al., 2008). Although there is no charge moved during the transition from the active

378 state to the relaxed state, the relaxed state can be detected through a slowing of the

379 gating kinetics as well as a left-shifted QV curve in R1C-qBBr:W454A;E247W and R1C-

380 qBBr:W454A;T326W (Figure 6-figure supplement 2). As with deactivation, qBBr

381 fluorescence can be used to visualize the relaxed state.

R1C-qBBr interaction with F290 provides the basis of the Cole-Moore shift

In addition to uncovering information about transitions to and from the resting,

385 active, and relaxed states, qBBr tracking provides novel insight into the Cole-Moore shift.

386 The Cole-Moore shift describes a phenomenon where following a prolonged

387 hyperpolarizing prepulse, a depolarizing pulse elicits a conductance that has a longer

388 time lag, or delay before beginning, than when there is no prepulse; this lag prolongs as

389 the prepulse is made longer and more negative (Cole \& Moore, 1960; Hoshi \&

390 Armstrong, 2015). The Cole-Moore shift is also seen in the gating currents underlying

391 the conductance (F. Bezanilla et al., 1994; Francisco Bezanilla et al., 1982). The Cole- 
392 Moore shift can be elicited in several ways. It is typically measured following a prepulse

393 of increasing hyperpolarized voltage before a depolarization (Figure 7A, top left) or

394 increasingly long hyperpolarizing prepulses (Figure 7A bottom left). The classical

395 explanation for the Cole-Moore shift is that a hyperpolarization populates closed states

396 that are further removed from the open state, thus delaying the opening as the channel

397 has to traverse more states before opening (Cole \& Moore, 1960). However, the

398 molecular basis of the Cole-Moore shift remains unknown (Hoshi \& Armstrong, 2015).

While measuring R1C-qBBr:W454A;F290W fluorescence, we observed no

400 appreciable fluorescence signal above the residual fluorescence in response to

401 depolarizing pulses from -120 mV (Figure 7A). However, in response to a

402 hyperpolarizing pulse to $-160 \mathrm{mV}$ from $-120 \mathrm{mV}$, we observed a small, markedly slow

403 reduction in fluorescence (Figure 7A). This fluorescence change did not have the same

404 kinetics of a small measurable gating current for the same pulse protocol (Figure 7-

405 figure supplement 1). When a protocol used to induce and measure a Cole-Moore shift

406 (Figure 7B, left bottom) was applied, the R1C-qBBr:W454A;F290W fluorescence

407 response was larger than the residual response seen in Fig. 7A, and it became slower

408 as the duration of the hyperpolarizing pulse increased (Figure 7C), as expected if this

409 fluorescence is associated with the Cole-Moore shift of the channel.

410 Previous studies have shown that at negative potentials R1C in the closed state can

411 spontaneously link to I287C, which is a full turn above F290 (Campos et al., 2007). Here,

412 we observe that at extreme hyperpolarizing potentials (-160 mV) R1C-qBBr moves near

413 F290W, producing a slow quenching of fluorescence. We explain this as a result of

414 moving the VSD with a strong hyperpolarization to such an extreme intracellular position 
A

C

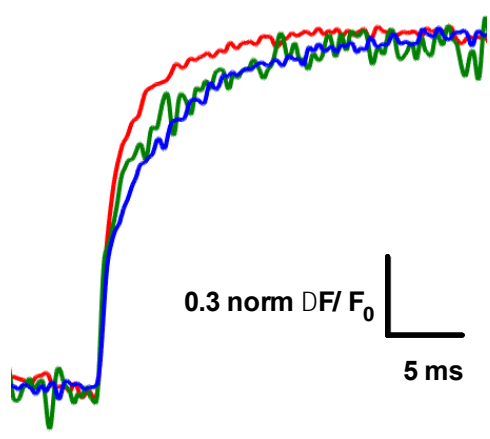

B

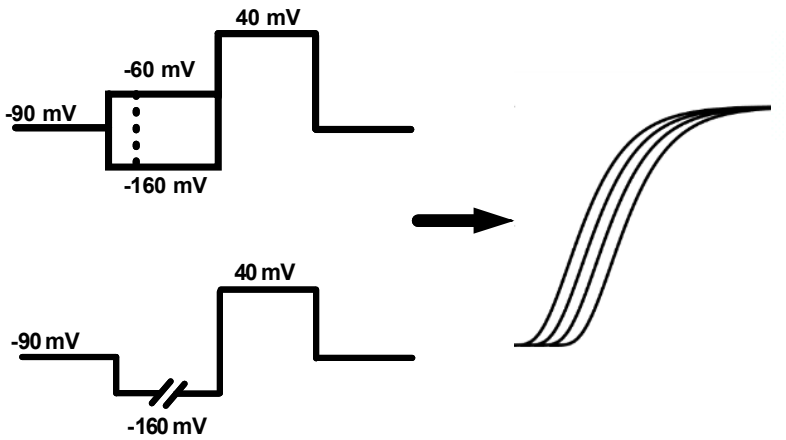

D

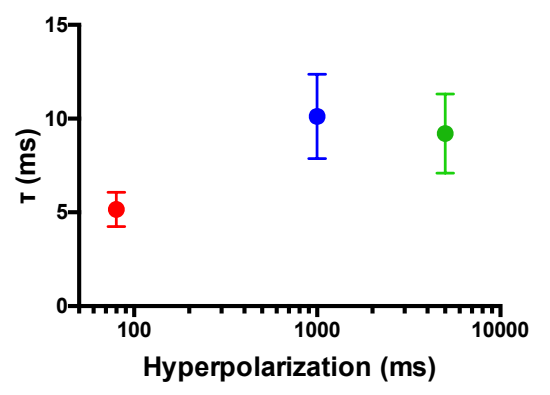

416 Figure 7. R1C-qBBr interaction with F290 provides the basis of the Cole-Moore shift

417 (A) A family of fluorescence traces for R1C-qBBr:W454A;F290W from a pulse protocol as in

418 (6A). Note the slow fluorescence response when hyperpolarized to $-160 \mathrm{mV}$ (purple) and the

419 residual signals during depolarizations. (B) Two pulse protocols that induce a Cole-Moore shift:

420 a variable voltage pulse before a depolarization (top) or a prolonged hyperpolarization pulse

421 before depolarization (bottom) and a representation of the resulting Cole-Moore shifts of the

422 ionic currents. (C) Representative normalized fluorescence traces for R1C-qBBr:W454A;F290W

423 using the pulse protocol from ( $\mathrm{B}$, bottom), with a $-160 \mathrm{mV}$ prepulse with a variable duration for 80

$424 \mathrm{~ms}$ (red), $1 \mathrm{~s}$ (blue), and $5 \mathrm{~s}$ (green). (D) Comparison of the $\boldsymbol{\tau}_{\text {weighted }}$ of the change in $\mathrm{qBBr}$

425 fluorescence in (C). (80 ms pulses, $n=4 ; 1$ s pulses, $n=5 ; 5$ s pulses, $n=4)$. Data are shown as

426 mean \pm SEM. 
428 that we populate other closed states of the voltage sensor that are responsible for the

429 Cole-Moore shift. These closed states are tracked by the fluorescence change

430 produced by an interaction between F290W and R1C-qBBr. A similar rarely observed

431 closed state predicted by metal-ion bridges, in which R1 transitions from a position

432 extracellular to F290 to a position intracellular to F290, was also suggested to provide a

433 potential explanation of the Cole-Moore shift (Henrion et al., 2012).

\section{Voltage-sensitive membrane protein CiVSP is interrogable by qBBr}

436 Our technique presented here allows for the interrogation of other VSD movements.

437 Another excellent candidate for qBBr mapping is CiVSP. With the reported crystal

438 structure (Li et al., 2014) we were able to begin qBBr mapping of this VSD (Figure 8A).

439 Using the CiVSP R217Q background (Dimitrov et al., 2007) we mutated the first gating

440 charge, R223, to a cysteine and labeled it with qBBr. The CiVSP R1C-qBBr showed

441 voltage-dependent fluorescence changes (Figure 8B, top). As with Shaker R1C-qBBr

442 we were able to identify the source of the fluorescent change. By mutating tyrosine

443 Y206 to an alanine, we abolished the voltage dependent fluorescence (Figure 8B,

444 bottom), while the voltage dependence of CiVSP with the Y206A mutation remained

445 intact (Figure $8 \mathrm{C}$ ). This result is a starting point for investigating the movement of the 446 gating charges of this VSD. 
A

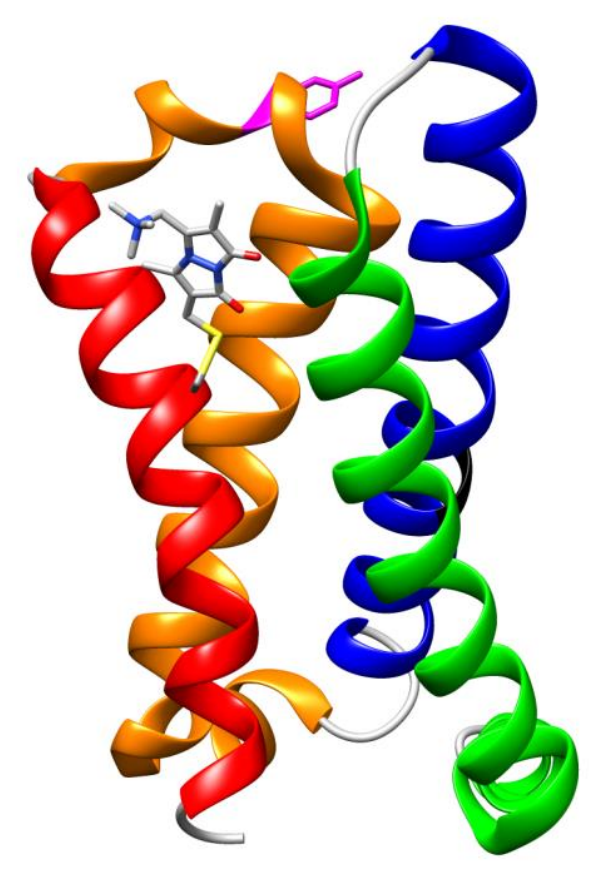

B
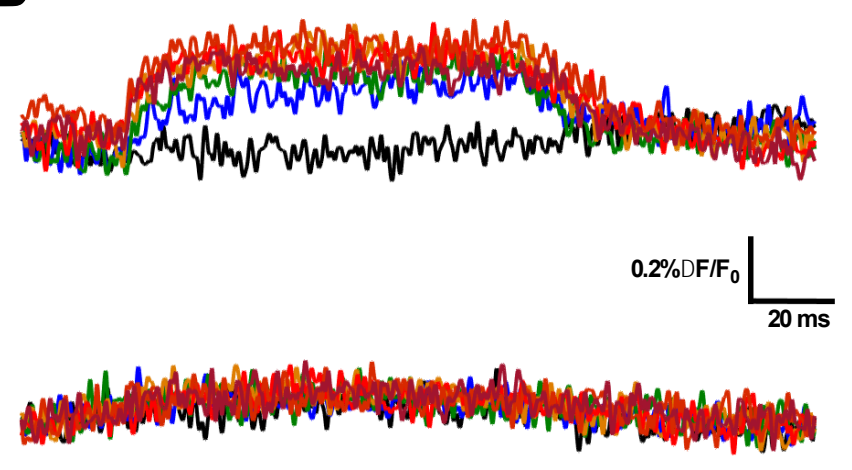

C

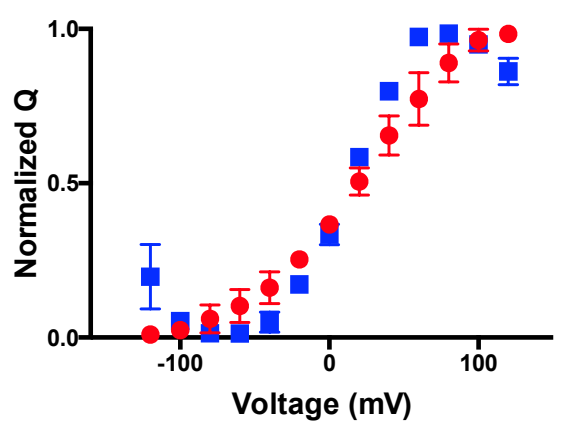

449 Figure 8. Voltage-sensitive membrane protein CiVSP is interrogable by qBBr

450 (A) CiVSP structure (PDB: 4G7V) with qBBr attached at R1C and highlighting residue Y206.

451 Transmembrane domains (S1-S4) are colored with S1, green; S2, blue; S3, orange; S4, red. (B)

452 Representative fluorescence traces of CiVSP R217Q R1C-qBBr (top) and CiVSP R217Q R1C-

453 qBBr:Y206A (bottom) (C) Normalized QV curves comparing CiVSP R217Q R1C-qBBr (red

454 circles, $n=4$ ) and CiVSP R217Q R1C-qBBr:Y206A (blue squares, $n=4)$. Data are shown as

455 mean \pm SEM.

457 Discussion

458 In this paper we have demonstrated a technique that allows tracking of a gating charge

459 surrogate in the pathway of a voltage sensor using qBBr. We have obtained new

460 information on the trajectories of the first two gating charges in Shaker (Figures 4 and 5). 
461 We propose that this is a flexible tool that should prove readily applicable to other

462 voltage-sensitive proteins. As we discuss the details of the trajectories, we will point out

463 some of the strengths and limitations of the technique.

464

\section{Spatial resolution}

466 In principle, one would expect qBBr mapping to be limited in its spatial resolution.

467 Tryptophan-induced quenching (TrIQ) and tyrosine induced quenching (TyrIQ) of

468 bimane dyes have been proposed as methods for measuring molecular distances

469 (Brunette \& Farrens, 2014; Mansoor et al., 2002, 2010). However, the maximum alpha

470 carbon to alpha carbon distance over which $\operatorname{TrlQ}$ occurs with qBBr has been found to

471 be around 10 to $11 \AA$ (Mansoor et al., 2010), or possibly as high as $15 \AA$ (Brunette \&

472 Farrens, 2014). It is clear that measurements of static distances with this method would

473 have poor spatial resolution, and it would seem that quenching over such long distances

474 would make it very difficult to trace any detailed pathway of qBBr. However, because we

475 are measuring a change in fluorescence as a function of time as the qBBr evolves in its

476 pathway, we obtain much higher spatial resolution than measuring static fluorescence

477 quenching in two positions. This is because we can detect very small changes in

478 relative fluorescence over time as the $\mathrm{qBBr}$ approaches or recedes from the quencher.

$479 \quad$ Take as an example qBBr moving from a position adjacent to $\mathrm{W}$ to a distance of

$4807 \AA$ away from the quencher during a depolarization. The qBBr-W distance is always

481 shorter than the $10 \AA$ known to be the qBBr-W quenching distance. However, the

482 degree of quenching will vary from extremely high when qBBr is close to W to less as it

483 moves to its final position. The change in fluorescence may be only 1 to $2 \%$, but this 
484 change still reveals that the qBBr-quencher distance is increasing. In other words, we

485 infer that it moved away even though we do not know the exact distance.

We cannot absolutely calibrate the degree of quenching, mainly because of

487 spurious background fluorescence. Therefore, the technique presented here takes full

488 advantage of the insertion of a quencher in the putative path: when a fluorescence

489 change occurs during a voltage pulse, we can infer the charge is moving with respect to

490 the quencher while if the signal does not change, it means that either the quencher is

491 far from the charge's path or there is no movement with respect to the quencher.

492 As a result, even though the interpretation is qualitative rather than quantitative,

493 our method not only constrains the resting and active positions of a particular gating

494 charge but also, by collecting data from many locations, generates a trajectory of the

495 transition pathway of the gating charge.

497 Kinetics of trajectories are different for each charge and depend on initial

498 conditions

499 As laid out in equation 2, in dynamic qBBr-W quenching, a two-state model

500 predicts that the kinetics of the fluorescence is independent of the position of qBBr with

501 respect to the quencher. If qBBr moves between several discrete positions, then

502 kinetics of the fluorescence is affected by the relative distance with respect to the

503 quencher (Equation 3). In a 3-state model the general prediction is a time course of

504 fluorescence with increases and decreases during the voltage pulse that moves the

505 charge. However, when the dwell time of the intermediate state is brief, then the

506 fluorescence change is monotonic and has two time constants. In all our studies, we do 
507 not see increases and decreases of fluorescence during a single pulse. Instead,

508 fluorescence changes are always in the same direction (either an increase or a

509 decrease), albeit with different kinetics and more than one time constant. The different

510 kinetics observed between different constructs, therefore, may be explained by the brief

511 dwell time in the intermediate state; however, we cannot exclude that the introduction of

512 a W mutation alters the underlying gating current kinetics. Consequently, although it

513 would be ideal to be able to compare kinetics of one construct to another, the possible

514 differences produced by the $\mathrm{W}$ mutations preclude us from doing so.

515 However, using the same construct, voltage pulses of different durations or

516 potential can be used to interrogate different transitions of the voltage sensor, and the

517 kinetics of different processes from the same construct can then be compared. For

518 example, the deactivation kinetics of the fluorescence change produced by R1C-

519 qBBr:W454A;T326W are more rapid than its activation kinetics, suggesting that the

520 interaction of $\mathrm{R} 1 \mathrm{C}-\mathrm{qBBr}$ with $\mathrm{T} 326 \mathrm{~W}$ is different during activation than during

521 deactivation. Additionally, we observe that the QV and FVs of deactivation have a

522 leftward shift to that of activation, thus demonstrating that the path of deactivation is

523 different than that of activation. This in turn informs us that the deactivation pathway of

$524 \mathrm{R} 1 \mathrm{C}-\mathrm{qBBr}$ must be different from its activation pathway, a conclusion that would be

525 difficult to obtain with any other method. Future studies could use qBBr optical mapping

526 to investigate the movement of discrete gating charges during conductive events to

527 correlate features of gating with ion conductance.

528 Another example is the slow R1C-qBBr:W454A;F290W fluorescence change

529 upon hyperpolarization. The kinetics of this interaction are markedly different from the 
530 kinetics of the main motion of the voltage sensor. This result gives direct evidence that

531 R1 can populate a region of the sensor closer to the intracellular side when the

532 membrane is strongly hyperpolarized and provides a molecular basis for the Cole-

533 Moore shift.

\section{Trajectories of discrete gating charges}

Our data indicate that in the normal resting state, it appears that R1 does not

537 come into close contact with F290 or 1294 in the S2 segment, while R2 does come into

538 proximity with 1294 . In the normal active state, R1 comes into close contact with W454

539 and $\mathrm{Y} 415$, while R2 comes into close contact with $\mathrm{Y} 415$, but does not interact with

540 W454. One observation potentially related to the position of the resting state is that two

541 of our tested constructs were lethal: R1C:W454A;I287W and R2C:F290W. Whether the

542 lethality of these constructs stems from a disruption of the normal resting state

543 interactions of discrete gating charges with the gating pore remains to be investigated.

544 However, R1 closely interacting with 1287 in the resting state is in good agreement with

545 earlier findings using disulfide bonding (Campos et al., 2007), fluorimetry (Pathak et al., 546 2007), and modeling (Henrion et al., 2012; Vargas et al., 2011).

547 The ability to optically track a gating charge in real time is a powerful technique,

548 despite its limitations. It allows for the combination of crystallographic studies and

549 functional data to understand the movement of the VSD. In general, our data with R1

550 and R2 supports a sliding helix model of movement of the gating charges but adds a

551 more detailed view of the movement. Each discrete gating charge undergoes both a

552 rotation with translation with an angle of about $60^{\circ}$ with respect to the plane of the 
553 membrane. Interestingly, the rotation of the R1 appears greater than the rotation of the

$554 \mathrm{R} 2$, suggesting that some of the movement may be coming from the movement of the

555 side chain, or a transient change of the helical conformation from an a helix to a 310

556 helix (Bassetto et al., 2020; Chakrapani et al., 2010; Henrion et al., 2012; Schwaiger et

557 al., 2011) rather than exclusively from the movement of the voltage sensor backbone.

558 We expect that this work can be expanded with molecular modeling. We may be able to 559 test whether the charges move independently of each other, with the first and second

560 gating charge moving first and then tugging the rest of the VSD along via the backbone 561 of the S4 segment (Horng et al., 2019).

563 position extracellular to F290 and indicate that interactions between this residue and R1

564 may be responsible for the Cole-Moore shift by moving the first charge deeper into the

565 intracellular side. This result is consistent with the shallow $\mathrm{Q}-\mathrm{V}$ curve recorded at very

566 negative potentials and also with the fact that the 'closed state' is in fact a collection of

567 closed states in agreement with the increase in entropy when evolving from the open to

568 the closed state (Claydon et al., 2007; Rodríguez et al., 1998).

570 Extension to other voltage sensors and gating charges

Numerous voltage sensors have been examined with site-directed fluorimetry:

572 Shaker (Cha \& Bezanilla, 1997; Mannuzzu et al., 1996), other Kv1-type channels

573 (Peters et al., 2009; Vaid et al., 2008), Kv7 (Kim et al., 2017; Osteen et al., 2010; Ruscic

574 et al., 2013), Kv10 and Kv11 (Schönherr et al., 2002; Smith \& Yellen, 2002), Nav (Cha

575 et al., 1999; Chanda \& Bezanilla, 2002; Wang et al., 2016), voltage-gated calcium 
576 channels (Pantazis et al., 2014), BK (Savalli et al., 2006), HCN (Bruening-Wright \&

577 Larsson, 2007), Catsper (Arima et al., 2018), and CiVSP (Kohout et al., 2008; Villalba-

578 Galea et al., 2008). Extension of the qBBr WY-induced quenching technique to other

579 voltage sensors is expected to provide new findings because this technique traces the

580 actual charge trajectories during voltage sensing. As a proof-of-principle, we

581 demonstrated qBBr can be applied to CiVSP, an evolutionarily distant voltage sensor.

582 Interestingly, we uncovered an interaction between R1 of CiVSP and a tyrosine residue

583 in the S3-S4 linker that is in good agreement with a reported crystal structure of the

584 voltage-sensing domain in the active state (Li et al., 2014). Further mapping of

585 additional voltage sensor pathways with the $\mathrm{qBBr}$ method described here should

586 improve our understanding of how voltage sensors and their discrete gating charges

587 move and provide new insight into where the electric field is focused, which side

588 charges interact with the VSD, and the differences in gating charge trajectories. Finally,

589 a present limitation of the technique is that $\mathrm{qBBr}$ mapping of gating charges is limited by

590 labeling accessibility to gating charge residues mutated to cysteine. For example, we

591 were unable to link $\mathrm{qBBr}$ to the third most extracellular Shaker gating charge (R368).

592 Future experiments using unnatural amino acid versions of $\mathrm{qBBr}$ or other fluorescent

593 amino acids with characteristics similar to qBBr (Leisle et al., 2015) may allow for the

594 expansion of this technique to additional charges in the voltage sensor.

596 Materials and Methods

597 Generation of constructs 
598 Mutations of Shaker or CiVSP DNA were made on the Shaker $\Delta 6-46$ W434F

599 background (Perozo et al., 1993) or the CiVSP C363S background (Murata et al., 2005),

600 respectively, using the QuikChange II site directed mutagenesis kit (Agilent, Santa Clara,

601 CA) with primers purchased from Integrated DNA Technologies. Shaker DNA was

602 linearized using Notl (New England Biolabs, Ipswich, MA) and CiVSP DNA was

603 linearized using Xbal (New England Biolabs); both were cleaned up with a NucleoSpin

604 Gel and PCR Clean-up kit (Macherey-Nagel, Bethlehem, PA). Shaker and CiVSP

605 cRNAs were then synthesized from linearized DNA using the mMESSAGE mMACHINE

606 T7 or SP6 transcription kit, respectively (Life Technologies, Carlsbad, CA).

Oocyte preparation

609 Oocytes were harvested from Xenopus laevis in accordance with experimental

610 protocols approved by the University of Chicago Animal Care and Use Committee.

611 Unless specified, all chemicals were obtained from Sigma-Aldrich (St. Louis, MO).

612 Following collagenase digestion of the follicular membrane, oocytes were maintained in

613 standard oocyte solution containing $96 \mathrm{mM} \mathrm{NaCl}, 2 \mathrm{mM} \mathrm{KCl}, 1 \mathrm{mM} \mathrm{MgCl} 2,1.8 \mathrm{mM}$

$614 \mathrm{CaCl}_{2}, 10 \mathrm{mM} \mathrm{HEPES}$, and $50 \mu \mathrm{g} / \mathrm{ml}$ of gentamicin, set with $\mathrm{NaOH}$ to $\mathrm{pH}$ 7.4, for up to

61536 hours prior to injection with $50 \mathrm{ng}$ of cRNA. Following cRNA injection, oocytes were

616 incubated at $16^{\circ} \mathrm{C}$ in standard oocyte solution for three to six days prior to recording.

618 Labeling with qBBr

619 The labeling solution consisted of depolarizing solution comprised of $120 \mathrm{mM} \mathrm{KCl,} 2$

$620 \mathrm{mM} \mathrm{CaCl}_{2}$ and $10 \mathrm{mM} \mathrm{HEPES}$ at $\mathrm{pH} 7.4$ with 1-2 mM qBBr (Sigma-Aldrich or Toronto 
621 Research Chemicals, North York, ON, Canada) added fresh to the solution upon each

622 preparation. Oocytes were maintained in the solution for at least fifteen minutes and

623 removed and washed in standard oocyte solution 5-20 minutes before recordings on

624 them were performed. At the longest, oocytes were maintained in labeling solution for

625120 minutes; however, due to the positive charge and impermeability of $\mathrm{qBBr}$, no

626 differences were observed between oocytes labeled for long durations versus short

627 durations.

Simultaneous fluorescence and electrophysiological recordings

630 Simultaneous electrophysiological and fluorescence recordings were performed at room

631 temperature using the cut-open oocyte voltage-clamp technique, similar to prior

632 descriptions (Cha \& Bezanilla, 1997; Lacroix et al., 2012; Villalba-Galea et al., 2008).

633 External solution contained $115 \mathrm{mM} \mathrm{N}$-methyl-D-glucamine, $2 \mathrm{mM} \mathrm{Ca}(\mathrm{OH})_{2}$, and $20 \mathrm{mM}$

634 HEPES taken to a pH between 7.4 and 7.5 with methanesulfonic acid. Internal solution

635 was like external solution, but with $2 \mathrm{mM}$ EGTA in place of $\mathrm{Ca}(\mathrm{OH})_{2}$. Pipettes were

636 pulled at a resistance of 0.2 to $1.0 \mathrm{M} \Omega$ and filled with $3 \mathrm{M} \mathrm{KCl}$. Excitation was performed

637 with a mounted $420 \mathrm{~nm}$ LED (ThorLabs, Newton, NJ) reflected by a $455 \mathrm{~nm}$ long-pass

638 dichroic (Chroma, Bellows Falls, VT) through a 40X water-immersion objective

639 (LUMPlan FL N, Olympus, Center Valley, PA); emission was collected through the

640 dichroic and a $475 \mathrm{~nm}$ long-pass filter (Chroma). Emission was integrated over each

641 sampling period through a home-built integrator, collected by a PIN-020A photodiode

642 (UDT Technologies, Torrance, CA), and amplified by a patch-clamp amplifier (L/M-EPC-

643 7, LIST Medical Electronics, Darmstadt, West Germany). Voltage-clamp and electrical 
644 measurements were performed with a CA-1B amplifier (Dagan, Minneapolis, MN). The

645 LED and voltage-clamp were controlled through Gpatch, an in-house acquisition

646 program, and an SB6711-A4D4 board (Innovative Integration, Simi Valley, CA).

647 Recordings at each voltage step were an average of four traces, taken consecutively, 648 sampled at $50-100 \mathrm{kHz}$ and filtered at $5-10 \mathrm{kHz}$.

Data analysis

651 Recordings were filtered and analyzed offline using custom MATLAB (Mathworks,

652 Natick, MA) scripts (Treger et al., 2015). Filtering of fluorescence for display and

653 analysis was performed using a digitized Bessel filter with a cutoff frequency between

$654500 \mathrm{~Hz}$ and $1 \mathrm{kHz} . \Delta \mathrm{F} / \mathrm{F}_{0}$ was calculated following a linear baseline subtraction of the

655 period 6 to $1.5 \mathrm{~s}$ prior to the electrical potential change of interest. Charge was

656 calculated from gating currents following linear baseline subtraction of user-defined

657 periods based on when gating current amplitudes returned to zero. Time constant

658 measurements of kinetics of both fluorescence and gating were calculated using either

659 a single exponential fit or a weighted double exponential fit to the rising or falling phase

660 of the fluorescence as appropriate, or to the decay component of the gating current.

$661 \quad$ Images of the Shaker and CiVSP proteins with qBBr and tryptophan substitutions

662 were created using UCSF Chimera (Pettersen et al., 2004).

663 
665 Statistical details of experiments can be found in figure legends. $\mathrm{N}$ represents the

666 number of oocytes, or biological replicates, and data are shown as mean \pm SEM.

667 Calculations were performed in Prism (GraphPad, La Jolla, CA).

668

References

670 Aggarwal, S. K., \& MacKinnon, R. (1996). Contribution of the S4 segment to gating charge in the Shaker K+ channel. Neuron, 16(6), 1169-1177. https://doi.org/10.1016/S0896-6273(00)80143-9

Ahern, C. A., \& Horn, R. (2004). Specificity of charge-carrying residues in the voltage sensor of potassium channels. The Journal of General Physiology, 123(3), 205216. https://doi.org/10.1085/jgp.200308993

Ahern, C. A., \& Horn, R. (2005). Focused electric field across the voltage sensor of potassium channels. Neuron, 48(1), 25-29. https://doi.org/10.1016/j.neuron.2005.08.020

Arima, H., Tsutsui, H., \& Okamura, Y. (2018). Conservation of the Ca2+-permeability through the voltage sensor domain of mammalian CatSper subunit. Channels (Austin, Tex.). https://doi.org/10.1080/19336950.2018.1476791

682 Baker, O. S., Larsson, H. P., Mannuzzu, L. M., \& Isacoff, E. Y. (1998). Three Transmembrane Conformations and Sequence-Dependent Displacement of the S4 Domain in Shaker K+ Channel Gating. Neuron, 20(6), 1283-1294. https://doi.org/10.1016/S0896-6273(00)80507-3 
686

687

688

689

690

691

692

693

694

695

696

698

699

700

701

702

703

704

705

Bassetto, C. A. Z., Carvalho-de-Souza, J. L., \& Bezanilla, F. (2020). Metal Bridge in S4 Segment Supports Helix Transition in Shaker Channel. Biophysical Journal, 118(4), 922-933. https://doi.org/10.1016/j.bpj.2019.08.035

Bezanilla, F., Perozo, E., \& Stefani, E. (1994). Gating of Shaker K+ channels: II. The components of gating currents and a model of channel activation. Biophysical Journal, 66(4), 1011-1021. https://doi.org/10.1016/S0006-3495(94)80882-3

Bezanilla, F., Taylor, R. E., \& Fernández, J. M. (1982). Distribution and kinetics of membrane dielectric polarization. 1. Long-term inactivation of gating currents. The Journal of General Physiology, 79(1), 21-40. https://doi.org/10.1085/jgp.79.1.21

Bezanilla, Francisco, White, M. M., \& Taylor, R. E. (1982). Gating currents associated with potassium channel activation. Nature, 296(5858), 657-659. https://doi.org/10.1038/296657a0

Broomand, A., Männikkö, R., Larsson, H. P., \& Elinder, F. (2003). Molecular movement of the voltage sensor in a K channel. The Journal of General Physiology, 122(6), 741-748. https://doi.org/10.1085/jgp.200308927

Bruening-Wright, A., \& Larsson, H. P. (2007). Slow Conformational Changes of the Voltage Sensor during the Mode Shift in Hyperpolarization-Activated CyclicNucleotide-Gated Channels. The Journal of Neuroscience, 27(2), 270-278. https://doi.org/10.1523/JNEUROSCI.3801-06.2007 
Brunette, A. M. J., \& Farrens, D. L. (2014, October 1). Distance Mapping in Proteins Using Fluorescence Spectroscopy: Tyrosine, like Tryptophan, Quenches Bimane Fluorescence in a Distance-Dependent Manner [Research-article]. https://doi.org/10.1021/bi500493r

710

Campos, F. V., Chanda, B., Roux, B., \& Bezanilla, F. (2007). Two atomic constraints unambiguously position the S4 segment relative to S1 and S2 segments in the closed state of Shaker K channel. Proceedings of the National Academy of Sciences, 104(19), 7904-7909. https://doi.org/10.1073/pnas.0702638104

Cha, A., \& Bezanilla, F. (1997). Characterizing voltage-dependent conformational changes in the Shaker K+ channel with fluorescence. Neuron, 19(5), 1127-1140. https://doi.org/10.1016/S0896-6273(00)80403-1

Cha, A., Ruben, P. C., George Jr., A. L., Fujimoto, E., \& Bezanilla, F. (1999). Voltage Sensors in Domains III and IV, but Not I and II, Are Immobilized by Na+ Channel Fast Inactivation. Neuron, 22(1), 73-87. https://doi.org/10.1016/S08966273(00)80680-7

Chakrapani, S., Sompornpisut, P., Intharathep, P., Roux, B., \& Perozo, E. (2010). The activated state of a sodium channel voltage sensor in a membrane environment. Proceedings of the National Academy of Sciences of the United States of America, 107(12), 5435. https://doi.org/10.1073/pnas.0914109107 
725 Chanda, B., \& Bezanilla, F. (2002). Tracking Voltage-dependent Conformational

726

727

728

729

730

731

732

733

734

735

736

737

738

739

740

741

742

743 Dimitrov, D., He, Y., Mutoh, H., Baker, B. J., Cohen, L., Akemann, W., \& Knöpfel, T.

744

Changes in Skeletal Muscle Sodium Channel during Activation. Journal of

General Physiology, 120(5), 629-645. https://doi.org/10.1085/jgp.20028679

Chen, X., Wang, Q., Ni, F., \& Ma, J. (2010). Structure of the full-length Shaker potassium channel Kv1.2 by normal-mode-based X-ray crystallographic refinement. Proceedings of the National Academy of Sciences, 107(25), 1135211357. https://doi.org/10.1073/pnas.1000142107

Claydon, T. W., Vaid, M., Rezazadeh, S., Kehl, S. J., \& Fedida, D. (2007). 4Aminopyridine Prevents the Conformational Changes Associated with P/C-Type Inactivation in Shaker Channels. Journal of Pharmacology and Experimental Therapeutics, 320(1), 162-172. https://doi.org/10.1124/jpet.106.110411

Cole, K. S., \& Moore, J. W. (1960). Potassium Ion Current in the Squid Giant Axon: Dynamic Characteristic. Biophysical Journal, 1(1), 1-14. https://doi.org/10.1016/S0006-3495(60)86871-3

Conti, L., Renhorn, J., Gabrielsson, A., Turesson, F., Liin, S. I., Lindahl, E., \& Elinder, F. (2016). Reciprocal voltage sensor-to-pore coupling leads to potassium channel C-type inactivation. Scientific Reports, 6, 27562. https://doi.org/10.1038/srep27562 (2007). Engineering and Characterization of an Enhanced Fluorescent Protein 
Voltage Sensor. PLOS ONE, 2(5), e440.

Henrion, U., Renhorn, J., Börjesson, S. I., Nelson, E. M., Schwaiger, C. S., Bjelkmar, P., Wallner, B., Lindahl, E., \& Elinder, F. (2012). Tracking a complete voltage-sensor cycle with metal-ion bridges. Proceedings of the National Academy of Sciences,

Hong, K. H., \& Miller, C. (2000). The Lipid-Protein Interface of aShaker K+ Channel. The Journal of General Physiology, 115(1), 51-58.

753

754

755

756

757

758

759

760

761

762

763

764

765 109(22), 8552-8557. https://doi.org/10.1073/pnas.1116938109

Horng, T.-L., Eisenberg, R. S., Liu, C., \& Bezanilla, F. (2019). Continuum Gating Current Models Computed with Consistent Interactions. Biophysical Journal, 116(2), 270-282. https://doi.org/10.1016/j.bpj.2018.11.3140

Hoshi, T., \& Armstrong, C. M. (2015). The Cole-Moore Effect: Still Unexplained? Biophysical Journal, 109(7), 1312-1316. https://doi.org/10.1016/j.bpj.2015.07.052

Islas, L. D., \& Zagotta, W. N. (2006). Short-range molecular rearrangements in ion channels detected by tryptophan quenching of bimane fluorescence. The Journal of General Physiology, 128(3), 337-346. https://doi.org/10.1085/jgp.200609556

Kim, R. Y., Pless, S. A., \& Kurata, H. T. (2017). PIP2 mediates functional coupling and pharmacology of neuronal KCNQ channels. Proceedings of the National Academy of Sciences of the United States of America, 114(45), E9702-E9711. https://doi.org/10.1073/pnas.1705802114 
766 Kohout, S. C., Ulbrich, M. H., Bell, S. C., \& Isacoff, E. Y. (2008). Subunit organization and functional transitions in Ci-VSP. Nature Structural \& Molecular Biology, 15(1), 106-108. https://doi.org/10.1038/nsmb1320

Labro, A. J., Lacroix, J. J., Villalba-Galea, C. A., Snyders, D. J., \& Bezanilla, F. (2012). Molecular mechanism for depolarization-induced modulation of $\mathrm{Kv}$ channel closure. The Journal of General Physiology, 140(5), 481-493.

Lacroix, J. J., \& Bezanilla, F. (2011). Control of a final gating charge transition by a https://doi.org/10.1085/jgp.201210817 hydrophobic residue in the $\mathrm{S} 2$ segment of a $\mathrm{K}+$ channel voltage sensor. Proceedings of the National Academy of Sciences, 108(16), 6444-6449. https://doi.org/10.1073/pnas.1103397108

Lacroix, J. J., \& Bezanilla, F. (2012). Tuning the voltage-sensor motion with a single residue. Biophysical Journal, 103(3), L23-25. https://doi.org/10.1016/j.bpj.2012.06.030

784 Lacroix, J. J., Labro, A. J., \& Bezanilla, F. (2011). Properties of deactivation gating 785 currents in Shaker channels. Biophysical Journal, 100(5), L28-L30. https://doi.org/10.1016/j.bpj.2011.01.043 
787 Lacroix, J. J., Pless, S. A., Maragliano, L., Campos, F. V., Galpin, J. D., Ahern, C. A.,

788 Roux, B., \& Bezanilla, F. (2012). Intermediate state trapping of a voltage sensor.

789 The Journal of General Physiology, 140(6), 635-652.

$790 \quad$ https://doi.org/10.1085/jgp.201210827

791 Lainé, M., Lin, M. A., Bannister, J. P. A., Silverman, W. R., Mock, A. F., Roux, B., \& Papazian, D. M. (2003). Atomic proximity between S4 segment and pore domain in Shaker potassium channels. Neuron, 39(3), 467-481. https://doi.org/10.1016/S0896-6273(03)00468-9 movement of the Shaker K+ channel S4. Neuron, 16(2), 387-397. https://doi.org/10.1016/S0896-6273(00)80056-2

Leisle, L., Valiyaveetil, F., Mehl, R. A., \& Ahern, C. A. (2015). Incorporation of NonCanonical Amino Acids. In C. Ahern \& S. Pless (Eds.), Novel Chemical Tools to Study lon Channel Biology (pp. 119-151). Springer New York. https://doi.org/10.1007/978-1-4939-2845-3_7 (2014). Structural mechanism of voltage-dependent gating in an isolated voltagesensing domain. Nature Structural \& Molecular Biology, 21(3), 244-252. https://doi.org/10.1038/nsmb.2768 
Mannuzzu, L. M., Moronne, M. M., \& Isacoff, E. Y. (1996). Direct physical measure of conformational rearrangement underlying potassium channel gating. Science, 271(5246), 213-216. https://doi.org/10.1126/science.271.5246.213

Mansoor, S. E., DeWitt, M. A., \& Farrens, D. L. (2010). Distance mapping in proteins using fluorescence spectroscopy: The tryptophan-induced quenching (TrIQ) Method. Biochemistry, 49(45), 9722-9731. https://doi.org/10.1021/bi100907m

Mansoor, S. E., Mchaourab, H. S., \& Farrens, D. L. (1999). Determination of Protein Secondary Structure and Solvent Accessibility Using Site-Directed Fluorescence Labeling. Studies of T4 Lysozyme Using the Fluorescent Probe Monobromobimane. Biochemistry, 38(49), 16383-16393. https://doi.org/10.1021/bi991331v

Mansoor, S. E., Mchaourab, H. S., \& Farrens, D. L. (2002). Mapping proximity within proteins using fluorescence spectroscopy. A study of T4 lysozyme showing that tryptophan residues quench bimane fluorescence. Biochemistry, 41(8), 24752484. https://doi.org/10.1021/bi011198i

McCormack, K., Joiner, W. I., \& Heinemann, S. H. (1994). A characterization of the activating structural rearrangements in voltage-dependent Shaker K+ channels. Neuron, 12(2), 301-315.

Menny, A., Lefebvre, S. N., Schmidpeter, P. A., Drège, E., Fourati, Z., Delarue, M., Edelstein, S. J., Nimigean, C. M., Joseph, D., \& Corringer, P.-J. (2017). 
Identification of a pre-active conformation of a pentameric channel receptor. ELife, 6, e23955. https://doi.org/10.7554/eLife.23955

829

830

831

Murata, Y., Iwasaki, H., Sasaki, M., Inaba, K., \& Okamura, Y. (2005). Phosphoinositide phosphatase activity coupled to an intrinsic voltage sensor. Nature, 435(7046), 1239-1243. https://doi.org/10.1038/nature03650

Osteen, J. D., Gonzalez, C., Sampson, K. J., Iyer, V., Rebolledo, S., Larsson, H. P., \& Kass, R. S. (2010). KCNE1 alters the voltage sensor movements necessary to open the KCNQ1 channel gate. Proceedings of the National Academy of Sciences, 107(52), 22710-22715. https://doi.org/10.1073/pnas.1016300108

Pantazis, A., Savalli, N., Sigg, D., Neely, A., \& Olcese, R. (2014). Functional heterogeneity of the four voltage sensors of a human L-type calcium channel. Proceedings of the National Academy of Sciences of the United States of America, 111(51), 18381-18386. https://doi.org/10.1073/pnas.1411127112

Pathak, M. M., Yarov-Yarovoy, V., Agarwal, G., Roux, B., Barth, P., Kohout, S., Tombola, F., \& Isacoff, E. Y. (2007). Closing in on the resting state of the Shaker K+ channel. Neuron, 56(1), 124-140. https://doi.org/10.1016/j.neuron.2007.09.023

Perozo, E., MacKinnon, R., Bezanilla, F., \& Stefani, E. (1993). Gating currents from a nonconducting mutant reveal open-closed conformations in Shaker K+ channels. Neuron, 11(2), 353-358. 
847 Peters, C. J., Vaid, M., Horne, A. J., Fedida, D., \& Accili, E. A. (2009). The molecular basis for the actions of KVß1.2 on the opening and closing of the KV1.2 delayed rectifier channel. Channels, 3(5), 314-322. https://doi.org/10.4161/chan.3.5.9558

Pettersen, E. F., Goddard, T. D., Huang, C. C., Couch, G. S., Greenblatt, D. M., Meng, E. C., \& Ferrin, T. E. (2004). UCSF Chimera-A visualization system for exploratory research and analysis. Journal of Computational Chemistry, 25(13),

Phillips, L. R., \& Swartz, K. J. (2010). Position and motions of the S4 helix during 1605-1612. https://doi.org/10.1002/jcc.20084

Priest, M., \& Bezanilla, F. (2015). Functional Site-Directed Fluorometry. Advances in Experimental Medicine and Biology, 869, 55-76. https://doi.org/10.1007/978-14939-2845-3_4

Rodríguez, B. M., Sigg, D., \& Bezanilla, F. (1998). Voltage Gating of Shaker K+ Channels The Effect of Temperature on lonic and Gating Currents. Journal of General Physiology, 112(2), 223-242. https://doi.org/10.1085/jgp.112.2.223 Goldstein, S. A. N. (2013). IKs channels open slowly because KCNE1 accessory subunits slow the movement of S4 voltage sensors in KCNQ1 pore-forming subunits. Proceedings of the National Academy of Sciences, 110(7), E559-E566. https://doi.org/10.1073/pnas.1222616110 
868 Savalli, N., Kondratiev, A., Toro, L., \& Olcese, R. (2006). Voltage-dependent conformational changes in human $\mathrm{Ca2}+-$ and voltage-activated $\mathrm{K}+$ channel, revealed by voltage-clamp fluorometry. Proceedings of the National Academy of Sciences, 103(33), 12619-12624. https://doi.org/10.1073/pnas.0601176103

872 Schönherr, R., Mannuzzu, L. M., Isacoff, E. Y., \& Heinemann, S. H. (2002). Conformational Switch between Slow and Fast Gating Modes: Allosteric Regulation of Voltage Sensor Mobility in the EAG K+ Channel. Neuron, 35(5), 935-949. https://doi.org/10.1016/S0896-6273(02)00869-3

Schwaiger, C. S., Bjelkmar, P., Hess, B., \& Lindahl, E. (2011). 310-Helix Conformation Facilitates the Transition of a Voltage Sensor S4 Segment toward the Down State. Biophysical Journal, 100(6), 1446-1454.

Semenova, N. P., Abarca-Heidemann, K., Loranc, E., \& Rothberg, B. S. (2009). Bimane fluorescence scanning suggests secondary structure near the S3-S4 linker of BK channels. Journal of Biological Chemistry, 284(16), 10684-10693. https://doi.org/10.1074/jbc.M808891200 in the S2 and S4 segments of the Shaker K+ channel. Neuron, 16(6), 1159-1167. https://doi.org/10.1016/S0896-6273(00)80142-7 
887 Smirnova, I., Kasho, V., \& Kaback, H. R. (2014). Real-time conformational changes in LacY. Proceedings of the National Academy of Sciences, 111(23), 8440-8445. https://doi.org/10.1073/pnas.1408374111

Smith, P. L., \& Yellen, G. (2002). Fast and Slow Voltage Sensor Movements in HERG Potassium Channels. The Journal of General Physiology, 119(3), 275-293. https://doi.org/10.1085/jgp.20028534

893

894

895

Starace, D. M., \& Bezanilla, F. (2001). Histidine Scanning Mutagenesis of Basic Residues of the S4 Segment of the Shaker K+ Channel. The Journal of General Physiology, 117(5), 469-490. https://doi.org/10.1085/jgp.117.5.469

Starace, D. M., Stefani, E., \& Bezanilla, F. (1997). Voltage-Dependent Proton Transport by the Voltage Sensor of the ShakerK+ Channel. Neuron, 19(6), 1319-1327. https://doi.org/10.1016/S0896-6273(00)80422-5

Tao, X., Lee, A., Limapichat, W., Dougherty, D. A., \& MacKinnon, R. (2010). A gating charge transfer center in voltage sensors. Science, 328(5974), 67-73. https://doi.org/10.1126/science.1185954

Treger, J. S., Priest, M. F., \& Bezanilla, F. (2015). Single-molecule fluorimetry and gating currents inspire an improved optical voltage indicator. ELife, 4, e10482. https://doi.org/10.7554/eLife.10482

Vaid, M., Claydon, T. W., Rezazadeh, S., \& Fedida, D. (2008). Voltage Clamp Fluorimetry Reveals a Novel Outer Pore Instability in a Mammalian Voltage- 
gated Potassium Channel. The Journal of General Physiology, 132(2), 209-222.

908 https://doi.org/10.1085/jgp.200809978

909

910

911

912

913

914

915

916

917

918

919

920

921

922

923

924

925

926

Vargas, E., Bezanilla, F., \& Roux, B. (2011). In search of a consensus model of the resting state of a voltage-sensing domain. Neuron, 72(5), 713-720. https://doi.org/10.1016/j.neuron.2011.09.024

Vargas, E., Yarov-Yarovoy, V., Khalili-Araghi, F., Catterall, W. A., Klein, M. L., Tarek, M., Lindahl, E., Schulten, K., Perozo, E., Bezanilla, F., \& Roux, B. (2012). An emerging consensus on voltage-dependent gating from computational modeling and molecular dynamics simulations. The Journal of General Physiology, 140(6), 587-594. https://doi.org/10.1085/jgp.201210873

Villalba-Galea, C. A., Sandtner, W., Starace, D. M., \& Bezanilla, F. (2008). S4-based voltage sensors have three major conformations. Proceedings of the National Academy of Sciences, 105(46), 17600-17607. https://doi.org/10.1073/pnas.0807387105

Wang, H.-G., Zhu, W., Kanter, R. J., Silva, J. R., Honeywell, C., Gow, R. M., \& Pitt, G. S. (2016). A novel NaV1.5 voltage sensor mutation associated with severe atrial and ventricular arrhythmias. Journal of Molecular and Cellular Cardiology, 92, 52-62. https://doi.org/10.1016/j.yjmcc.2016.01.014

Yang, N., \& Horn, R. (1995). Evidence for voltage-dependent S4 movement in sodium channels. Neuron, 15(1), 213-218. 
bioRxiv preprint doi: https://doi.org/10.1101/2020.04.23.058818; this version posted April 24,2020 . The copyright holder for this preprint (which was not certified by peer review) is the author/funder, who has granted bioRxiv a license to display the preprint in perpetuity. It is made available under aCC-BY 4.0 International license.

927 Yao, X., Parnot, C., Deupi, X., Ratnala, V. R. P., Swaminath, G., Farrens, D., \& Kobilka,

928 B. (2006). Coupling ligand structure to specific conformational switches in the $\beta 2-$

929 adrenoceptor. Nature Chemical Biology, 2(8), 417-422.

$930 \quad$ https://doi.org/10.1038/nchembio801

931 


\section{Supplemental Figures}

A

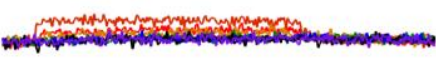

B

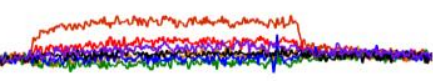

C

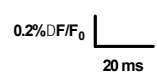

Figure 2-figure supplement 1. R1C-qBBr:W454A and R2C-qBBr fluorescence signals are not produced by endogenous tryptophans or tyrosines Representative fluorescence traces of qBBr labeled (A) R1C-qBBr:W454A;W289F (B) R1C-qBBr:W454A;Y323F (C) R2C-qBBr W289F;Y323F. In all panels, membrane potentials during the pulse are: brown, $+80 \mathrm{mV}$; red, $+40 \mathrm{mV}$; orange, $0 \mathrm{mV}$; green, -40 $\mathrm{mV}$; blue, $-80 \mathrm{mV}$; black, $-120 \mathrm{mV}$; purple, $-160 \mathrm{mV}$. 
A

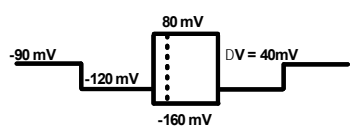

B

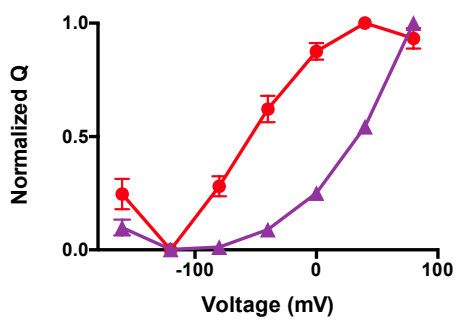

C

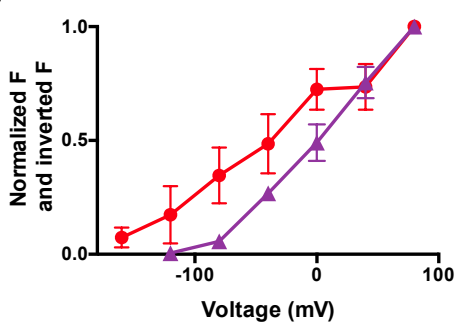

D

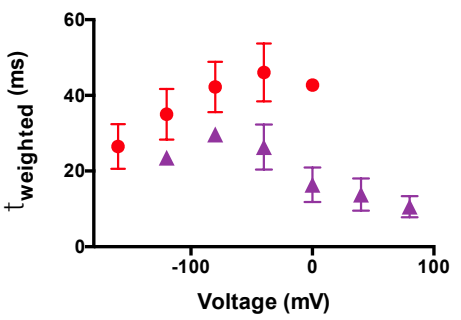

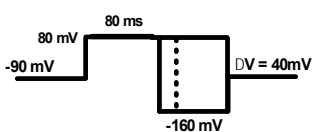

I287W
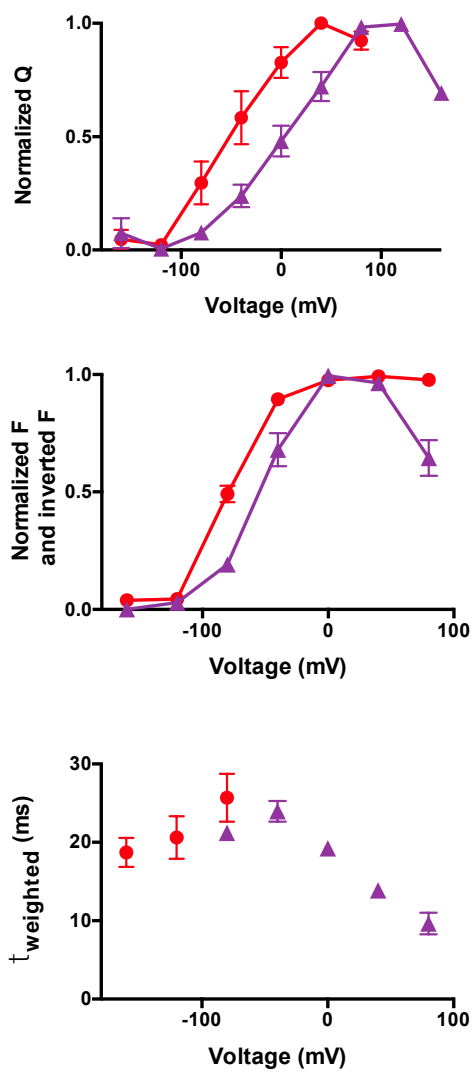

A Activation

- Deactivation

\section{Y415W}
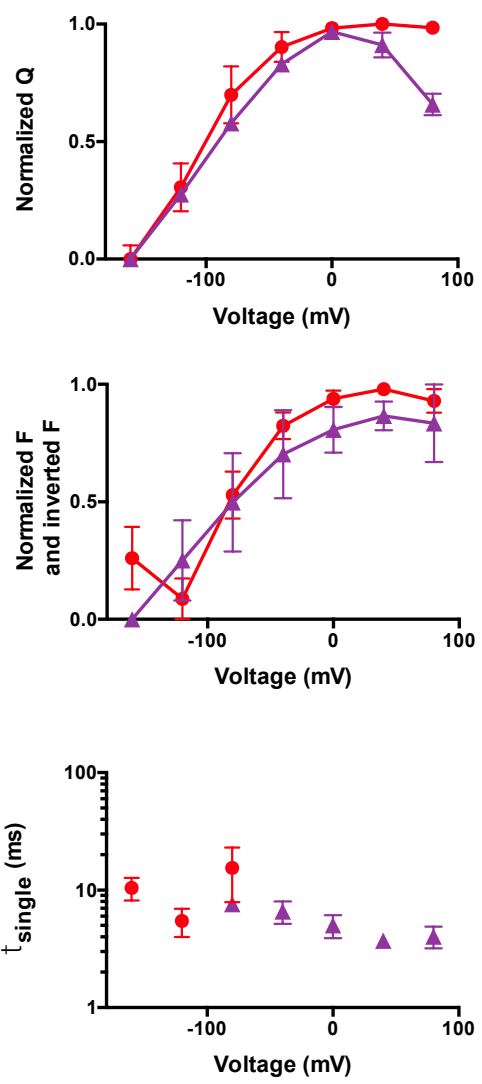

Figure 6-figure supplement 1. The VSD deactivation path of R2C-qBBr differs from that of activation

(A) Activation (purple) and deactivation (red) protocols. Shading indicates where gating and fluorescence were measured for (B-D). (B) Comparison of activation (purple triangles) and deactivation (red circles) QVs for R2C-qBBr:F244W (left, n=4), R2CqBBr:I287W (center, $n=5$ ), and R2C-qBBr:Y415W (right, $n=4$ ). (C) As in (B), but a comparison of activation and deactivation FVs, rather than QVs. (D) Comparison of single exponential $\boldsymbol{\tau}_{\text {act }}$ (red circles, $n=4$ ) to $\boldsymbol{\tau}_{\text {dea }}$ (purple triangles, $n=7$ ) of R2CqBBr:F244W (left), weighted $\tau_{\text {act }}$ (red circles, $n=4$ ) to $\boldsymbol{\tau}_{\text {dea }}$ (purple triangles, $n=5$ ) of R2CqBBr:I287W (center), and weighted $\boldsymbol{\tau}_{\text {act }}$ (red circles, $n=5$ ) to $\boldsymbol{\tau}_{\text {dea }}$ (purple triangles, $n=4$ ) of R2C-qBBr:Y415W (right). Data are shown as mean \pm SEM. 
A

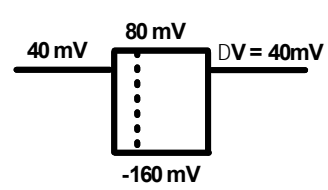

B

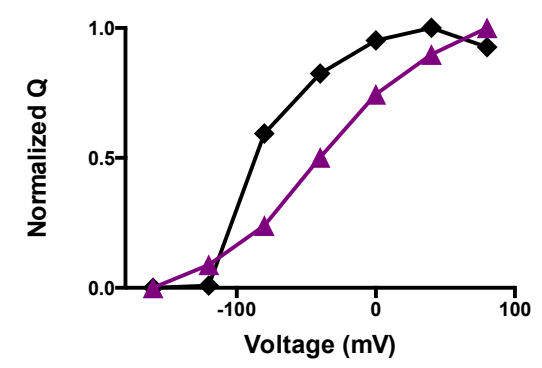

C

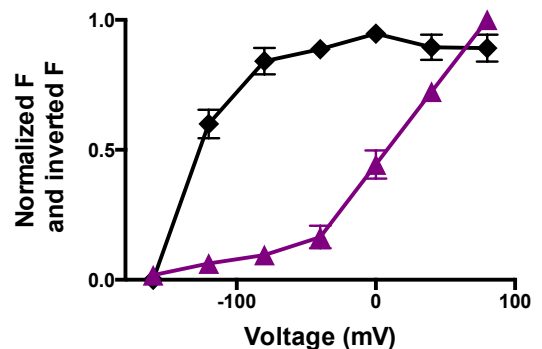

$\leftarrow$ Activation

$\leftrightarrow$ Relaxed

T326W
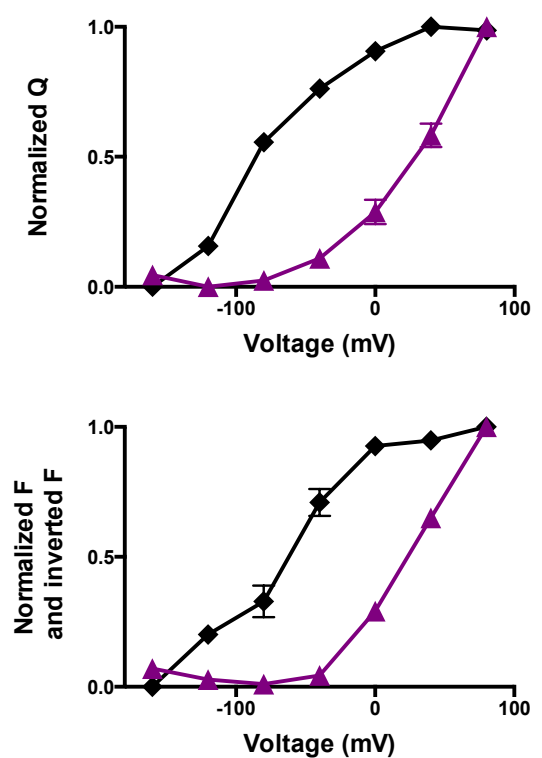

Figure 6-figure supplement 2. R1C-qBBr fluorescence visualizes the relaxed state. (A) A relaxation pulse protocol. (B) Comparison of activation (purple, triangles) and derelaxation (black, diamonds) QVs for R1C-qBBr:W454A;E247W (left, $\mathrm{n}=4$ ) and R1C-qBBr:W454A;T326W (right, $n=4$ ). (C) Inverted $F V_{\text {normalized }}$ and $F V_{\text {normalized for }}$ activation and derelaxation for R1C-qBBr:W454A;E247W (left, activation $\mathrm{n}=8$, derelaxation $n=6$ ) and R1C-qBBr:W454A;T326W (right, activation $n=5$, derelaxation $\mathrm{n}=6$ ). Data are shown as mean \pm SEM. 


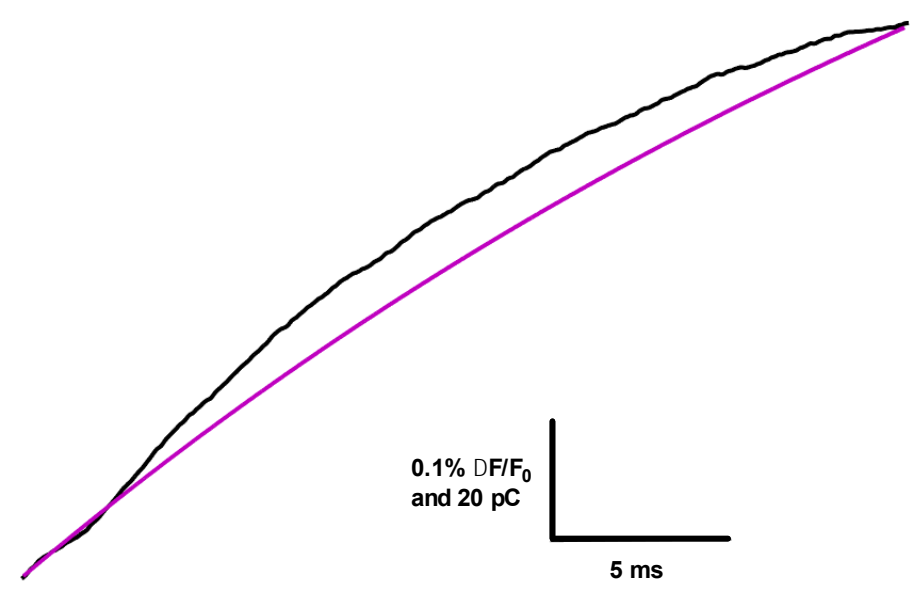

Figure 7-figure supplement 1. R1C-qBBr:W454A;F290W hyperpolarizationinduced gating charge and fluorescence signal time course

Data is taken from a pulse from $-120 \mathrm{mV}$ to $-160 \mathrm{mV}$. The integrated gating current of all four charges moving through the electric field (gating charge, $Q$, black) is more rapid than the hyperpolarization-induced fluorescence signal of R1C-qBBr:W454A;F290W, shown as the single exponential fit to the inverted fluorescence signal (purple). The fluorescence signal only follows the movement of the first charge. Traces are aligned to each other, but the fluorescence signal is still slower than the gating charge. 